\title{
Report to Congress on Server and Data Center Energy Efficiency: Public Law 109-431
}

\author{
Appendices \\ ENVIRONMENTAL ENERGY TECHNOLOGIES DIVISION \\ Ernest Orlando Lawrence Berkeley National Laboratory \\ University of California \\ Berkeley, California 94720
}

August 2007 


\section{EPA REPORT TO CONGRESS ON SERVER AND DATA CENTER ENERGY EFFICIENCY}

\section{APPENDICES}

Appendix 1. Glossary and Definition of Key Terms ....................................................... 1

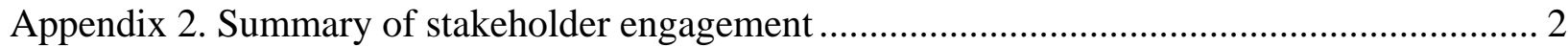

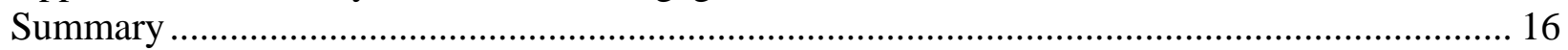

Appendix 3. Fuel Cell Installations in Data Centers and Related Premium Power Applications 27

Case Studies of Combined Heat and Power Applications at Data Centers ............................ 29

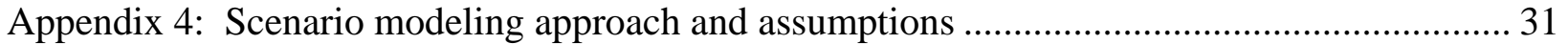

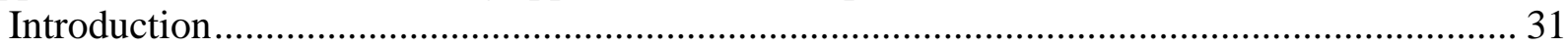

Modeling Approach and Assumptions for the Historical Trends and Current Efficiency Trends

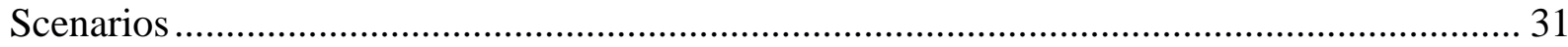

Modeling Approach and Assumptions for the Additional Efficiency Scenarios.................... 40

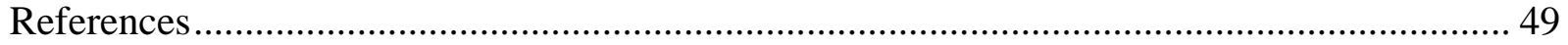

Appendix 5. Summary of current state energy efficiency programs ..................................... 51 


\section{Appendix 1. Glossary and Definition of Key Terms}

Benchmark: to evaluate by comparison to a standard. In the context of energy efficiency, benchmarking involves measuring the energy performance of a product or building by means of a standard metric, e.g., kWh of annual energy use per square foot of building floor area. The measured performance value can then be compared to the performance of similar products or buildings.

Electrostatic: Pertaining to static electricity.

High-end servers: Defined by market research firm IDC as servers with an average sales value of $\$ 500,000$ or more.

Hygroscopic: A substance that readily attracts and retains water.

Infrastructure equipment: All equipment in a building outside of the IT equipment racks, such as the HVAC system, PDUs, UPSs, and building lighting.

Mid-range servers: Defined by market research firm IDC as servers with an average sales value of $\$ 25,000$ to $\$ 499,999$.

Power density: Power of a given set of equipment divided by a given area of floor space. Confusion often arises when discussing power use in data centers if these terms are not accurately defined. Three power density terms are used throughout this report and defined below.

Computer power density: Power drawn by the computer equipment divided by the computer room floor area.

Building power density: Total power drawn by the building divided by the total floor area of the building.

Total computer room power density: Power drawn by the computer equipment and all supporting equipment such as PDUs, UPSs, HVAC, and lighting divided by the computer room floor area.

Power usage effectiveness: Defined as the ratio of total data center energy use to total IT equipment energy use.

Relative humidity: an index of the water content of air, expressed as a percentage of the maximum amount of water the air can hold at that temperature.

Server consolidation: The consolidation of multiple applications on fewer hardware-based servers.

Virtualization: Virtualization is an abstraction layer that decouples the physical hardware from the operating system. Virtualization allows multiple virtual machines, with heterogeneous operating systems to run in isolation, side-by-side on the same physical machine.

Volume servers: Defined by market research firm IDC as servers with an average sales value below $\$ 25,000$. 


\section{Appendix 2. Summary of stakeholder engagement}

To develop this report, EPA convened a study team led by researchers from the Lawrence Berkeley National Laboratory. The study team offered stakeholders multiple opportunities to give input to and review this report, including:

- conducting preliminary calls with key stakeholders to help plan the study;

- $\quad$ holding a public workshop on February 16, 2007 (attended by approximately 130 people) to solicit input on the topic of energy efficiency in servers and data centers;

- following up on workshop attendees' offers of assistance, to gather and refine information for the study;

- posting on the ENERGY STAR web site an open call for interested parties to submit information, as well as a list of data needs;

- posting on the ENERGY STAR web site a public review draft of this report; and

- incorporating into the final version of this report more than 50 sets of comments on the public review draft.

Several documents produced for and resulting from the February 2007 public workshop are included here:

a. Workshop agenda.

b. Workshop working group outcome summary notes

c. Identified data needs for stakeholder input

d. List of workshop attendees 


\title{
Workshop agenda
}

\author{
EPA Technical Workshop on Energy Efficient Servers and Datacenters in the U.S. \\ February 16, 2007 \\ Santa Clara Convention Center \\ Rooms 209/210
}

Purpose: To work with industry stakeholders in developing a work plan for EPA's study of energy efficiency opportunities in servers and datacenters and to identify ways that industry and other stakeholders can collaborate and assist with this study.

\section{8:15 - 8:30 am \\ 8:30 am}

9:00 am

9:30 am

10:00 am

10:15 am

10:30 am

10:30 - 12:00 pm

$12: 00 \mathrm{pm}$

12:15 - 1:45 pm

\section{Registration and Breakfast}

Welcome and Introduction - Andrew Fanara, U.S. EPA.

- Introduction and workshop goals

- EPA's interest in servers and datacenters

- Overview of HR 5646 and introduction of EPA Study Team

- $\quad$ ENERGY STAR roadmap for servers and datacenters

\section{Current State of Knowledge: Server and Datacenter Energy Use - Jonathan} Koomey, Ph.D., Stanford University

Overview of EPA Study - Eric Masanet, Lawrence Berkeley National Laboratory

- Summary of work plan and interpretation of study requirements

- Vision for final report

- Purpose and goals of working group sessions

\section{Q\&A}

Break

Attendees Break into Working Group Sessions Group Topics: (1) IT Equipment; (2) Power and Cooling Infrastructure; (3) Integrated Design, Operation, and Management Issues; (4) Incentives and Voluntary Programs

Morning Session - Each group will be presented with a task summary and work plan for discussion. Attendees begin outlining information gaps.

Working Lunch - Attendees pick up lunch in the main discussion room and return to working groups to continue focused discussions.

Afternoon Session - Each group will define process and available resources to address information gaps in the study, to be reported back to the larger group.
$1: 45 \mathrm{pm}$
Break
2:00 pm
Summary of Working Group Sessions - Task Leads
$3: 00$ pm
Discussion of Results \& Information Sharing Between Groups
$3: 30 \mathrm{pm}$
Wrap-Up: Next Steps and Action Items - Andrew Fanara, U.S. EPA
4:00 pm
Adjourn 


\section{Workshop working group outcome summary notes}

\section{Summary Notes for Working Group 1 (IT Equipment)}

\section{A: Estimation of growth trends and trends in IT equipment energy use}

Topics of discussion:

- Deriving estimates of growth trends utilizing existing data sources Ideas generated:

- Use historical data on IT equipment shipments from IDC

- Try to understand key demand behavior that is fundamentally driving growth

- Must understand trends toward increasing consolidation/virtualization

— Must understand trends related to utilization

- ASHRAE Power Trends might offer useful information

- Consider looking to trends on data transactions as proxy for growth (perhaps large users such as banks, healthcare, etc. can help)

- Consider looking at trends in shipments of power supplies as a proxy for the trends in shipments of servers (a company will typically spend $2 \%$ of server budget on power supplies)

- Deriving estimates of power use by servers, storage devices, and network equipment Ideas generated:

— Peak versus idle power by component might be important to consider

- Koomey study results and approach for server energy use seem reasonable

- Consider characterizing trends that show that energy performance is improving while total power consumption is going up due to demand increasing faster

- Historical facility benchmark data may be useful for understanding trends

- Data on energy use breakdown among servers, network, and storage devices is needed, but it is not clear where such data exist

- Consider the effects of equipment redundancy (for reliability) when estimating energy use

- For network equipment, the dynamic range (idle to peak) of power use is small; thus, it is more important for existing equipment to understand how many network devices are connected than to understand utilization

- Consider surveying IT managers or service providers on how they provision networks

- Determine Federal vs. non-Federal split for installed base of IT equipment Ideas generated:

— Perhaps data on Federal sales are available from large server vendors 


\section{B: Definition of plausible efficiency scenarios and estimation of cost savings}

Topics of discussion:

- Estimation of future energy use (5 years out) for several plausible future scenarios Ideas generated:

- Three possible scenarios seemed to resonate with the working group:

1. Business as usual (BAU): a scenario based on projecting current trends in energy use, sector growth, equipment end uses, rates of utilization, etc. This is the "no policy intervention" scenario.

2. Best practice: a scenario in which all best available technologies and management strategies are employed to reduce the energy use of IT equipment in the data center. This scenario examines what could be done with currently available technologies and management expertise if they were implemented across the board.

3. Emerging technologies: a scenario in which technologies currently in development but expected to hit market in the next five years are examined. This scenario captures the energy efficiency benefits of the next wave of technology.

- A key future trend to consider is efficiency improvements at the chip level

- Low adoption rates are a key barrier to moving from the BAU scenario to the best practice scenario even though more energy efficiency technologies have been available for years; finding ways to overcome this barrier will be key for recommendations

- The use of virtualization and power management are important trends to capture

- Data are needed on energy use and trends in energy efficiency for all IT equipment, not just servers and microchips as stated in the H.R. 5646 text

- Power supply efficiency trends also need to be considered

- Consider in the scenario analyses that businesses that experience higher power growth trends are more likely to adopt power saving technologies

\section{C: Identification and discussion of reliability and performance issues}

Topics of discussion:

- Identification of potential impacts of energy efficiency on reliability, performance, cost, and speed

Ideas generated:

— Consider that, in general, by adding more complexity to a system (for example, using power management software) more points of failure are added to a system

— Thermal conditions are key to IT equipment reliability, thus reliability is tied to HVAC issues 
- Many current practices for reliability lead to redundancy and thus to higher power consumption

- There is a need to dispel the notion that more energy efficient equipment is less reliable because this isn't the case in many operations and this myth is a persistent barrier to improving the efficiency of data centers

- MTTR (mean time to repair) might be an important metric to capture

- Performance "hits" might not matter as long as they are aligned with services that can absorb such "hits"

— Data on reliability versus number of parts are available, which may help

\section{D: Recommendations regarding potential incentives and voluntary programs} Topics of discussion:

- Identification and discussion of possible recommendations for incentives and voluntary programs

Ideas generated:

- Financial incentives (tax credits, energy efficiency rebates) could be built into the cost of IT equipment so that extra work by the end user is not needed to claim the credit/rebate

- Labels like ENERGY STAR can be effective both for providing the manufacturer with an incentive and for educating the end use customers on the benefits of lower energy use

— IT managers should be better educated on the cost benefits of energy efficiency

- Financial incentives could be awarded to the manufacturer then passed along to the consumer

- Verification of whether or not end users are using power management features is key (but difficult) for seeing if savings are actually realized

- The SPEC benchmark could be a useful metric for promoting energy savings

- Federal procurement policies can go a long way since the government is such a large customer

- Any metrics that are used to characterize energy efficiency must be designed carefully and should encompass performance

\section{Summary Notes for Working Group 2 (Data Center Infrastructure)}

\section{A: Growth and efficiency trends, market segmentation, and potential cost savings}

The legislation directly dictates segmenting federal vs. non-federal. Segmenting into other markets such as institutional and size are also implied. A proper desegregation of the data center market is of one of the first challenges in this evaluation. This requires defining a data center and potential sub-categories. The suggested parameters to define and categorize data centers are as follows: 


\section{Definition of Data Center (defining characteristics):}

Separate HVAC

LBNL defines a data center as, "a room that has an independent HVAC zone,"

independent of size. This means the data center could be an entire building or simply a closet with a dedicated HVAC unit.

Emergency Backup Power

Data centers typically have backup power, though this is not always the case.

Raised Floor

Data centers typically have raised floors, though this is not always the case.

\section{Security}

Data centers typically have enhanced security

\section{Building Codes}

There are specific codes that already define an IT room or data center. The two codes suggested were the National Electric Code, Article 645; and NFPA 75

\section{Categorization of Data Centers}

Floor Area

The Uptime Institute categorizes data centers into different size tiers by "electrically active" floor area. It was suggested that the performance of the infrastructure may vary significantly be size. The LBNL study, however, did not see a correlation with size, Bus Quantity though the study the study did not include any very small (closet size) data centers.

Major data centers have dual bus applications, thus the UPS cannot run better than $50 \%$ load. Non-critical data centers only have a single UPS or single bus and can run at higher efficiencies.

\section{Power Demand}

It was suggested that categorizing the size data centers by the power draw $(\mathrm{kW})$ of the IT equipment would be more accurate than floor area.

\section{Cover Groups/Over-provisioning}

Bill Kosik, from EYP, did a paper on data center dynamics that separated data center operations in enterprise cover groups and search engine cover groups.

Federal vs. Non-Federal

Boston Sullivan and Venture Development Corporation (VDC) should have some data on this.

New vs. Old Data Centers

It is not clear which is more efficient. New centers have newer equipment, but they are also oversized for anticipated future loads.

\section{Estimating Power Consumption of Data Centers}

Jon Koomey's study estimated the power consumption by estimating the critical load from servers (based on server sales) and then using a total power/critical load ratio.

One of the goals of this group is to confirm the Koomey analysis. One strategy could be to take Koomey’s data for IT equipment Koomey's estimates, and then estimate the appropriate total power/critical load ratio to use for different efficiency scenarios.

The following total power/critical load ratios were proposed: 


\begin{tabular}{|l|l|l|l|l|}
\hline $\begin{array}{l}\text { Benchmark } \\
\text { Source }\end{array}$ & $\begin{array}{l}\text { Ideal Best } \\
\text { Practice }\end{array}$ & $\begin{array}{l}\text { Actual Best } \\
\text { Practice }\end{array}$ & Typical & Worst \\
\hline $\begin{array}{l}\text { Uptime } \\
\text { (from two papers) }\end{array}$ & 1.6 & 1.8 & $2.4-2.6$ & $3.2-3.5$ \\
\hline LBNL & 1.2 & 1.3 & 2.0 & 3.0 \\
\hline
\end{tabular}

\section{Ratio Adjustments}

The above ratios will be modified as more data are received (potential data sources are described below). Ideally, different ratios will be applied to each data center category

\section{Ratio Trends} (i.e. big, small, federal, non-federal).

Many felt the ratio would change over time, strictly due to market forces and with no change in policy. The reasons given for this change are:

1. Increased interest in energy efficiency would cause the ratio to drop

2. The change in ratio would be geographically dependent as certain technologies favor specific climates.

3. Data center consolidation would drop the ratio as many small centers are sold and new large centers are built (large centers as considered more efficient and new centers are now built with efficiency in mind)

4. The ratio may increase as centers more to areas with cheaper electricity

5. The ratio may increase due to increase redundancy. In the last five years there has been increased redundancy due to IT availability (more redundancy causes increased electrical power)

Overall, the group felt the base case ratio will probably drop by about $2 \%$ per year, with no change in policy. No change in ratio could be expected for the worst case scenario

\section{Identified Data Sources}

LBNL (above table)

Data may be skewed towards better data centers due to self-selection.

The Uptime Institute (above table)

Data comes from two studies, measured data representing the total power to run the data center, always running refrigeration (no economizers) and includes both member and non-members of Uptime.

Intel

They have no large compiled set of data, but they have talked with customers to understand where energy is being used.

IDC Reports:

Leibert:

Source of Jon Koomey’s study.

MW

Has 79 different data points on CRAC units. Can be split out from 0.5MW to about 8

Representative from Midwest [needs to be identified] 
Involved with study that estimate 1-2 million square feet of raised floor (broken out data centers and office space)

Bert and Turner

Gave a presentation last year using the following categorizations:

\begin{tabular}{|l|l|l|}
\hline Size Category & Floor Area & Installed Servers \\
\hline Small & $15,000 \mathrm{ft}^{2}$ & $300-500$ \\
\hline Medium & $20,000 \mathrm{ft}^{2}$ & $1500-1700$ \\
\hline Large & $35,000 \mathrm{ft}^{2}$ & N/A \\
\hline Very Large & $>100,000 \mathrm{ft}^{2}$ & N/A \\
\hline
\end{tabular}

\section{Side Note}

Monitoring Equipment

While the critical load is easy to measure, determining the load required to operate a data center can be difficult to obtain. Installed monitoring equipment may be the first step in increasing efficiency: it allows the data center to better understand their relative performance. Such an approach can also be used for reliability and predictive maintenance.

\section{B: Impact on Electric Grid}

This part of the report involves converting the energy savings estimated in the previous task to a peak load savings (using the National Energy Modeling System). To do this, we need to understand where the load savings will occur in the grid.

Peak Load Trends

The load is becoming more of a curve than a flat line, due to efficiency measures (economizers, weather) and project schedules (engineers submitting batch jobs on Friday to run over the weekend, rendering being performed at night)

While overall contribution to energy demand may be small ( 1.2\%), impact may be significant at certain location.

Migration Trends:

More data center consolidation in the Pacific Northwest due to cheap power. This power is cheap because of underutilized generation capacity, but prices will go up as capacity is used up.

The state of Montana is developing energy plans, expecting an increased demand once the rates in eastern Washington begin to increase.

Potential Solutions

Distributed Generation could alleviate peak demand, but a standby connection is always required, which is very expensive.

Thermal Energy Storage

\section{C: Non energy impacts of improved efficiency}

Reduced Cost

Potential downsizing due to efficient equipment

Lower maintenance

More efficient systems can delay having to add another facility Increased Reliability

Running UPS at high efficiency levels extends the lives of electrical systems 
Fewer hotspots. More cooling equipment capacity results in a reduced indoor thermal quality (it can be difficult to properly control air as capacity increases, resulting in hotspots)

More consistent temperature. Reduced reliability associated with higher temp and greater variations. Temperature changes cause the expansion/contraction of metals, and cause server fans to ramp up. It may reduce the life, but the question is: is it enough to matter, relating to the savings in the operational cost

Ergonomics

Warmer cold isles will be more comfortable for data center occupants

Hotter hot isles will be unpleasant for data center occupants

Reduced noise

Improved Competitiveness

Increased capacity

Stimulated Economy

Retrofits

\section{D: Benefits of distributed generation/cogeneration (e.g. fuel cells) DG/CHP Key Issue:}

1. Electricity generation and heat (boiler) on-site: efficiency can get into $70 \%$.

2. Point that makes sense "spark spread"

3. Utility interconnect

4. Reliability: redundancy can be avoided, the grid could be used as backup, but standby charges (access to grid) is very high

5. Incentives to produce power when utility needs it

6. More complicated systems, new unfamiliar business, and data center users are wary to relinquish control to $3^{\text {rd }}$ party providers

7. Regulatory issues (air quality)

8. Lack of critical mass, track record (concern for response during an emergency)

9. Education/training, O\&M reliability

10. Fuel cell waste heat not at high enough quality (some technology)

11. Current fuel cell technology efficiency is about $37 \%$ (includes reform), though some of the emerging solid oxide technologies are believed to achieve substantially high efficiencies

12. Turbines are more common

\section{E. Incentives, voluntary programs, $R \& D$, and industry activities Key Barriers}

1. Risk of new/different technologies

2. Inadequate monitoring

3. Huge standby charges 
4. Lack of knowledge

5. Split incentives (different budgets), IT doesn't pay utility bills

6. Lack of information (lower temp of range always chosen)

7. Mature design/build (old school mentality)

8. First cost

9. Required redundancy, conservative marketplace (risk to owner and risk to A\&E)

10. ROI varies with change in energy prices (uncertainty in cost of fuel)

11. Short time horizon, IT vs. facility

12. Other management issues

\section{Working Group Recommendations}

1. Incentives to manufacturers

2. Building or finance DG

3. Energy star for HVAC equipment

4. LEED for data centers

5. Incentives for efficient systems and operations

6. Education training: owner/operators, designers (show value through assessment programs)

7. Government investment demonstration projects: showcase cutting edge technologies with case studies (see which ones fail, succeed). For example Canadian government only buys efficient equipment

8. Incentives for monitoring

9. Technology development: R\&D, demo, incentives

10. Test standards and ratings: COP, total/IT

11. Top down approach: get the CEO/CIO on board

12. Government could reduce "load" on data center

13. Roadmap with target goals (coming up with technically feasible goals)

14. Roadmap performance tied to (tax) incentives (i.e. set efficiency target for UPS, then government will purchase an initial amount)

15. Work with ASHRAE, IEEE, Green Grid

16. Greater utilization of all component without reducing reliability (incentive to remove legacy equipment)

17. R\&D: Improve cooling chain, power chain, address from a system approach, computational output

18. Incentives to increase utilization and virtualization 


\section{Summary Notes for Working Group 3 (Integrated Design, Operation, and Management}

\section{Issues)}

\section{Disconnect between IT and facilities}

- IT pays their power bill by square foot, not by power consumption -> make users pay true cost

- integration of responsibility for construction and operations (integrate IT and facilities)

- better planning: mismatch between installed capacity and actual loads (overbuilding)

- defining business purpose is difficult

- create better incentives for people in charge of buying servers (right now they don't care about buying efficient servers)

- organization matters

- convert facilities requirements into business goals (buy efficient/more expensive servers, b/c we'll pay less for energy in the long term)

- integrate design and operations

- standard model for total cost of ownerships of servers

- not just cost of server, but space, power, cooling, operations

- understand the dependency of software (how much power is my application using?)

- what should we measure?

- rack, server power consumption

- real time

- capacity utilization

- infrastructure

- total power/IT load

- what to report? what are the right metrics?

- power and thermal management go hand in hand

- standardization of airflow to allow hot/cold aisle

- waste heat

- low utilization of servers

- different departments don't share the equipment

\section{Risk will often trump energy efficiency!}

- heroics $=$ risk 
- data centers require certain temperature and humidity, but do servers really fail more often when running outside these regions?

- organizational issues:

- real estate

- facilities

- procurement

- IT

- power/influence

- demonstration projects/case studies in federal facilities

\section{Data from Gartner Data center conference (show of hands, 2000 people)}

- 60 - 70\% will be replaced in the following 5 years because they don't support high densities

- 90\% of data centers will be virtualizing

\section{Smaller working groups (before lunch):}

\#1: organizational issues

\#2: information issues: standards + metrics

\#3: time/planning issues

\#4: everything else

\section{\#1: organizational issues:}

1. risk. vs. reward vs. simplification

- ROI (what's the reward of spending extra money on efficient servers)

- uptime is still most important

2. cost allocation (need to understand the real cost)

- need to measure power consumption, efficiency

- come up w/ metrics to drive decisions

- capex vs. opex

3. IT and facilities gap

- people who are in charge of buying servers have no incentive to buy efficient servers

4. optimizing the system redundancy

- what's the risk vs. reward?

- but what's really risky? we can make better measurements, better metrics

- often the energy bill is not significant (compared the other expenses)

- but this is only when the data center is small 
- some colo's charge by kWh (instead of square feet)

- IT data centers have very low utilization

- web services data centers have high utilization (MS -> 80 - 90\% utilization)

- lack of communication inside of company

- express everything in \$ (easy to understand)

- need to measure stuff before we can express it in $\$ \$$

\section{\#2: information (lack of):}

1. metrics and benchmarking

- metrics of IT equipment

- IT power vs. total power

- cooling vs. total power

- true DC utilization

2. information sharing between departments

- requirements to report efficiency

- end to end analysis when planning the infrastructure

- social responsibility (report CO2 emissions)

3. incentives

- tax incentives

- incentives to adopt new technology

- market for CO2

4. need for quantitative education

\section{\#3: time/planning}

1. metrics for performance, reliability and efficiency for all components and operations - to mitigate risk of adoption of new technology

2. organizational processes and structure to address integrated planning and operations - success depends on people working together (integrated planning)

3. segmentation: addressing incentives for different people working in a company - one size doesn't fit all

\section{\#4: risk and everything else}

1. integrated full life-cycle risk model

- risk increases when we add dynamic provisioning

- no quantitative way to model the risk and economic effects

- how do you add capacity as needed

- how do you structure SLAs?

2. demonstration projects: 
- insufficient sharing of best-practice

- best-practices become corporate IP

- ??: who will take the first step to build the first product and share with the community

3. TCO:

- good and complete model of TCO (umbrella)

\section{Smaller working groups (after lunch):}

1. metrics/visibility

- IP issues?

- measure improvements, also absolute

- business continuity

- separate IT metrics from infrastructure metrics

- learn from green building movement

2. new models of computing

- utility computing model

- dynamic workloads

- network detached computing

3. analysis of systemic investment

- grid

- assessment of costs and benefits

4. life-cycle risk assessment

- learn from green building movement (bring all designers to the table)

- need integrated architecture metrics (include storage and networks)

\#1: metrics/visibility

- metrics

- I: tax

- integrated data center infrastructure design

- get seal of approval if you follow a process

- education

- certificates for engineers/operators

- demonstration projects

- creating federal DC demo project (central test lab to stimulate innovation

- federal funding of university project

\section{\#2: new models of computing}

- metrics and benchmarking

- integrated \& segmented b/m

- public reporting

- standards and best practices 
- IT components, facility components

- integrated DC design

- certificates

- optimizing redundancy

- incentives

- external: work with utilities

- internal: tool \& practices to facilitate decision making

- education

- tool that would help with metrics

\#3: analysis of systemic investment

- blind comparison of metrics

- life-cycle risk assessment

- best practices w/o risking IP

\section{Summary Notes for Working Group 4 (Incentives and Voluntary Programs)}

\section{Summary}

At the end of the working group session, the facilitators pulled together an outline summarizing the key points brought up by the working group for the purposes of reporting to the afternoon general session:

1. 3 Key positive drivers
a. \$ savings
b. Environmental savings (carbon, etc.)
c. Grid relief - fewer blackouts

2. Key desired outcomes

3. Then show barriers and policies to overcome these barriers

a. Top down, education and awareness

4. Top down approach (i.e., initiative must come from upper management) needed to overcome barriers

a. Corporate / government challenge for data center efficiency - praise and shame

b. Government can set an example

c. Standard metric is key - must be supplier independent

d. Top 100 List of most efficient data centers

e. Money is key

i. Demonstrate the business case

ii. Financial incentives for efficient data centers (tax incentives and utility incentives)

iii. The right decision maker needs to see the incentive

5. Awareness / Education / Certification / Training

a. Educate sustainability officers within the organization using the data center

b. "Carbon police"

c. Audits

d. Government case studies 
6. Research and Development (R\&D)
a. Demos
b. X-prize challenge
c. Data center test lab

7. Harmonized utility programs - several utilities across a region coordinating their programs for data center efficiency

8. Industry standards

9. Focus on areas of great change - volume servers, federal facilities

10. "No CIO Left Behind" program - incentives for achievement in data center efficiency 11. Structure:

a. Desired outcome - e.g., $90 \%$ or greater power supply efficiency

b. Policies to achieve this

\section{Key points of discussions on incentives and voluntary programs captured during the} working group session:

1. Market segments
a. Own vs. lease
b. Care about energy efficiency or not
c. Only care if power/cooling problems
d. Carbon goals - environment is a driver

2. Over provision - education needed

3. Energy concern driven by power/cooling constraints

a. More data centers in this group

4. Forecasting compute and power/cooling requirements
a. Metrics
b. Dashboard tools

5. Data centers located in a larger building

a. How to meter/benchmark?

6. Need education and incentives to get better estimates of compute load

7. Coordination between IT and facilities

8. Facilities learn how IT equipment works

a. IT heat loads

b. More sophisticated cooling

9. Technical education needed to be improved

10. Personal risk - e.g., dead servers don't get turned off

11. Why don't managers track server utilization / data IO?

a. No credit for efficiency

b. Key thing is rolling out new apps and keeping them running

12. Demand-based switching
a. $10 \%$ implementation

13. Organizational change needs to be top-down (starts with CIO)

14. Efficient solution is a risk
a. Have leaders demo new technologies
b. Sell cases where energy efficiency reduces risk (outside air)
c. Government leadership 
d. Small innovators - small firms

15. Drivers (top down)

a. Save money

b. Savings carbon

c. Grid reliability

16. Energy efficiency tie-in to other issues

a. Sprawl

b. Manageability

17. Key is reducing \# of servers

18. Small firms - someone has responsibility for entire problem

a. Can be more innovative

19. Need someone in the organization who understands energy issues

20. CIO Magazine - energy costs are a large part of total cost of ownership (TCO)

21. IT doesn't measure energy, but facilities department does

a. How to create a model where IT personnel see the energy price?

22. Energy is not cheap - it's a major part of TCO now

23. Don't forget infrastructure energy use

a. Incentive for running data center at better IT/infrastructure ratio

24. What to do with legacy data centers?

25. Problem is not the components, it's how they are used

26. Concerns (drivers)
a. Heat density
b. TCO
c. Carbon cap

27. Incentives:

a. Technical audits (performance contracting)

i. Need follow-up over time

b. DOE program doing energy audits

c. Tax credit for investment

28. Different situations - greenfield, expansion, existing data center retrofit

29. Low or no cost - audit

30. Meter data centers and do benchmarking

31. Shorten payback

32. Develop customer demand for energy efficiency (e.g., Wal-Mart)

33. Incentive for efficient servers

34. Benchmark site infrastructure

a. Government endorsement for companies to do this

35. Data center rating and certification

a. Malcolm Baldridge Quality award

b. LEED

c. Recognition for improvement in energy efficiency rating

36. Harmonized utility programs

a. National SBC

37. Get unused equipment turned off

a. servers ship with power management turned on

38. Need three things for successful energy efficiency 

a. Awareness
b. Capability
c. Motivation

39. Government can identify a metric for benchmarking

a. Separate benchmark for IT and infrastructure

40. Influence $\mathrm{CIO} / \mathrm{CFO}$

a. Government case studies

41. Government procurement

a. Use energy efficiency as a factor in selecting data center contractors

42. Educate corporate environment / responsibility officer

a. Sustainability - DJ sustainability index

43. Top 100 energy efficiency organizations in CIO magazine - sponsored by EPA

44. Tax credits get CFO attention

45. Carbon credits

46. Industry-harmonized set of metrics (what to measure and report)

47. AEE certification for IT efficiency

48. Basic technical education for data center operators

49. Auditor for training - 1000 data center Challenge

50. National Data Center Test Lab

51. Federal government IT installations

a. Demonstrate benchmarking

b. Challenge private sector to match federal

c. Enforcement of federal procurement rules

i. Anecdote about VERY inefficient servers at SLAC

52. Sponsor data center upgrade to best practice efficiency

\section{Identified data needs for stakeholder input}

\section{Summary of Identified Data Needs}

The data needs listed below were identified at the February $16^{\text {th }}$ Technical Workshop at the Santa Clara Convention Center based on working group discussions. The list was sent to workshop participants and also posted on the ENERGY STAR website. The study team welcomed all information sources and leads from interested stakeholders that could help address the data needs listed in the six categories below.

\section{1: Estimation of growth trends in IT equipment and data centers}

Historical data (2000 - present) and projected data (over next 5 years) are needed for:

- Installed base and shipments of servers, storage devices, and network equipment

$0 \quad$ By end use sector (Federal vs. non-Federal)

o By end use category (data center vs. workgroup computing)

o By U.S. region

o By equipment class (e.g., volume vs. high-end servers, tape vs. hard disk drives, routers vs. switches) 
ـ Trends in IT equipment utilization (average \% of peak capability)

a Trends in virtualization

a Floor area of U.S. data centers

a Trends in underlying demand for data services fueling server computing and data center growth in the United States

a Trends in data center computing density (W/ $/ \mathrm{ft}^{2}$, etc.)

\section{2: Estimation of energy use by IT equipment and data centers and analysis of trends toward more efficient components and servers}

Historical data (2000 - present) and projected data (over next 5 years) are needed for:

- Energy used by servers

o By server class

o Idle to peak load energy use relationship

o Projected trends based on component and server efficiency improvements

a Microchip energy use trends

口 Power supply energy efficiency trends

- Energy used by storage devices

o By type of storage device

o Idle to peak load energy use relationship

o Projected trends based on energy efficiency improvements

- Energy used by network equipment

o By type of network device

o Projected trends based on energy efficiency improvements

\ Benchmark data on total data center energy use

口 Total data center energy use/IT equipment energy use ratios

o Ideally, broken down further by IT equipment type

- Key trends in energy use of infrastructure systems (power conversion, backup power, cooling, etc.)

3: Estimation of cost savings due to improved IT equipment and data center energy efficiency 
- Quantitative and qualitative information (including case studies) on non-energy related cost savings and benefits (e.g., improved performance, reduced capital expenditures, etc.) of improved energy efficiency

4: Analysis of the potential cost savings and benefits to the energy supply chain through the adoption of energy efficient data centers and IT equipment

u Utilization and power load shapes for various types of data center operations

a Regional breakdown of server and data center operations

\section{5: Analysis of the potential impacts of energy efficiency on product performance}

a Quantitative and qualitative information (including case studies) on potential positive and negative impacts of energy efficiency on product performance, including computing functionality, reliability, speed, and features, and overall cost

6: Analysis of the benefits of the use of distributed generation (DG)/cogeneration (e.g., CHP)/fuel cells

- Quantitative and qualitative information on industry experience with DG/CHP, the perceived role of DG/CHP, the perceived benefits/barriers/issues, power reliability requirements, reliability strategies and approaches, and current use and cost of back-up power systems 


\section{List of workshop attendees}

\begin{tabular}{|c|c|c|c|}
\hline Companyl Organization & Last Name & $\begin{array}{l}\text { First \& Middle } \\
\text { Names }\end{array}$ & Title \\
\hline 365 Main, Inc. & Balajadia & Jean-Paul & SVP Operations \\
\hline Active Power & Perkins & David E. & Chief Technology Officer \\
\hline AMD & Kerby & Brent & Manager of Commercial Business \\
\hline AMD & Rawson & Andrew & $\begin{array}{l}\text { Senior Member Technical Staff, } \\
\text { Advanced Server System Archetect }\end{array}$ \\
\hline AMD & Sadowy & Donna & SMTS \\
\hline $\begin{array}{l}\text { American Power } \\
\text { Conversion }\end{array}$ & Bean & John & Director, R\&D - Cooling \\
\hline $\begin{array}{l}\text { American Power } \\
\text { Conversion }\end{array}$ & Carlini & Steve & \\
\hline $\begin{array}{l}\text { American Power } \\
\text { Conversion }\end{array}$ & Dunlap & Kevin & Director, Business Strategy - Cooling \\
\hline $\begin{array}{l}\text { American Power } \\
\text { Conversion }\end{array}$ & Sharp & Glenn & Enterprise Acct. Manager \\
\hline $\begin{array}{l}\text { American Power } \\
\text { Conversion }\end{array}$ & Tuccillo & John & \\
\hline ANCIS Incorporated & Herrlin & Magnus K. & President \\
\hline Astec Power & Phadke & Vijay & Sr.Technical Fellow \\
\hline Astec Power & Hannon & Rich & Senior Engineering Fellow \\
\hline Austin Energy & Johnson & Anne & Engineer \\
\hline Austin Energy & Noriega & Michelle & $\begin{array}{l}\text { Conservation Program Specialist, } \\
\text { Senior }\end{array}$ \\
\hline Bay Area PowerXperts & Kuczer & Alan & \\
\hline Bloom Energy & Eggers & Matt & \\
\hline $\begin{array}{l}\text { California Data Center } \\
\text { Design Group }\end{array}$ & Greco & Richard A. & \\
\hline $\begin{array}{l}\text { California Energy } \\
\text { Commission }\end{array}$ & Kulkarni & Pramod & Manager- Industrial Energy Efficiency \\
\hline $\begin{array}{l}\text { California Energy } \\
\text { Commission }\end{array}$ & Roggensack & Paul & Mechanical Engineer \\
\hline Capricorn Technologies & Spampinato & Janice & Acting VP, Sales and Marketing \\
\hline Cisco Systems & Broer & Andy & $\begin{array}{l}\text { IT Manager } \\
\text { NextGen Production Data Center } \\
\text { Design \& Build }\end{array}$ \\
\hline Cisco Systems & Naheem & Sheikh & \\
\hline Cisco Systems & Poon & Daisy & $\begin{array}{l}\text { Eco-Design Standards Technical } \\
\text { Leader } \\
\text { Corporate Compliance }\end{array}$ \\
\hline Cisco Systems & Russo & Joe & Sr. Eng. Mgr. Service Provider \\
\hline $\begin{array}{l}\text { Clustered Systems } \\
\text { Company }\end{array}$ & Hughes & Phil & CEO \\
\hline
\end{tabular}




\begin{tabular}{|c|c|c|c|}
\hline $\begin{array}{l}\text { Critical Facilities Round } \\
\text { Table }\end{array}$ & Myatt & Bruce C. & Founder \\
\hline CSRware & Alonardo & Karen & Founder and CEO \\
\hline CSRware & Salem & Krimo & Founder and CTO \\
\hline Degree Controls, Inc. & Phelps & Walter & Data Center Product Manager \\
\hline Dell & Pflueger & John & $\begin{array}{l}\text { Technology Strategist } \\
\text { Office of the CTO }\end{array}$ \\
\hline Dell & Taylor & Jay & \\
\hline Delta Products Corp. & Hunter & Graham & Vice-President, Sales \\
\hline $\begin{array}{l}\text { Dennis Peck and } \\
\text { Associates }\end{array}$ & Peck & Dennis & Principal \\
\hline DOE & Scheihing & Paul & $\begin{array}{l}\text { Team Leader, Technology Delivery } \\
\text { Industrial Technologies Program }\end{array}$ \\
\hline Eaton Electrical Corp. & Giangrosso & Patrick L. & $\begin{array}{l}\text { Dir. of Business Development - Mission } \\
\text { Critical Facilities }\end{array}$ \\
\hline Eaton Electrical Corp. & Wallace & Ian & Senior Specialist \\
\hline eBay, Inc. & Lee & Tim & Manager, Data Center Asset Control \\
\hline eBay, Inc. & Reder & Libby & Program Manager, Global Citizenship \\
\hline eBay, Inc. & Santana & Paul & Sr Manager, Site Services, Sacramento \\
\hline EMC & Winkler & Kathrin & $\begin{array}{l}\text { Sr. Director, Product Management } \\
\text { Common Storage Platform Operations }\end{array}$ \\
\hline Emerson Network Power & Miller & Robert J. & $\begin{array}{l}\text { Vice President } \\
\text { Marquee Accounts }\end{array}$ \\
\hline EPA & Fanara & Andrew & \\
\hline EPRI & Fortenbery & Brian & Program Manager \\
\hline Fairchild Semiconductor & Laumeister & Bill & \\
\hline $\begin{array}{l}\text { Fujitsu Siemens } \\
\text { Computers }\end{array}$ & Henning & Bernd S. & $\begin{array}{l}\text { Director Technology Strategy and } \\
\text { Innovation }\end{array}$ \\
\hline $\begin{array}{l}\text { Fujitsu Siemens } \\
\text { Computers }\end{array}$ & Reger & Jospeh & СТО \\
\hline General Electric & Hall & Nickolas & Sourcing Leader-Midrange \\
\hline Google & Barroso & Luiz & Distinguished Engineer \\
\hline Google & Weihl & Bill & Energy Strategy \\
\hline Green Grid & Tipley & Roger & \\
\hline GreenIT & Hodges & Richard & Principal \\
\hline $\begin{array}{l}\text { Hewlett-Packard } \\
\text { Company }\end{array}$ & Belady & Christian & Distinguished Technologist \\
\hline $\begin{array}{l}\text { Hewlett-Packard } \\
\text { Company }\end{array}$ & Goldstein & Martin & \\
\hline
\end{tabular}




\begin{tabular}{|c|c|c|c|}
\hline $\begin{array}{l}\text { Hewlett-Packard } \\
\text { Company }\end{array}$ & Malone & $\begin{array}{l}\text { Christopher } \\
\text { Gregory }\end{array}$ & Thermal Technologies Architect \\
\hline $\begin{array}{l}\text { Hewlett-Packard } \\
\text { Company }\end{array}$ & Olinger & Bill & \\
\hline Hitachi America Ltd. & Obata & Toshinori & Product Manager \\
\hline Hitachi Data Systems & Logan & Dave & VP Planning \\
\hline Hitec Power Solutions & Sears & John & Marketing/Sales Manager \\
\hline IBM & Brey & Tom & \\
\hline IBM & Dietrich & Jay & $\begin{array}{l}\text { CEA Program Manager: Climate } \\
\text { Stewardship }\end{array}$ \\
\hline IBM & Keller & Tom & \\
\hline IBM & Prisco & Joe & $\begin{array}{l}\text { IBM Senior Systems and Technology } \\
\text { Group Engineer }\end{array}$ \\
\hline ICF International & Buchwalter & Sarah & Research Assistant \\
\hline ICF International & Duff & Rebecca & Project Manager \\
\hline ICF International & Haines & Evan & Research Assistant \\
\hline ICF International & Hedman & Bruce & Vice President \\
\hline IDC & Wu & Jie & Senior Research Analyst \\
\hline Intel & Hengevald & Jon & \\
\hline Intel & Patterson & Michael & \\
\hline Intel & Rego & Chuck & \\
\hline Intel & Wigle & Lorie & \\
\hline Intel & Wong & Henry M & \\
\hline $\begin{array}{l}\text { Jones Lang LaSalle } \\
\text { Americas }\end{array}$ & Ali & Syed L. & $\begin{array}{l}\text { Global Energy Manager for the Sun } \\
\text { Microsystems Account Jones Lang } \\
\text { LaSalle Americas, Inc. }\end{array}$ \\
\hline LBNL & Brown & Rich & \\
\hline LBNL & Koomey & Jonathan & $\begin{array}{l}\text { Staff Scientist at Lawrence Berkeley } \\
\text { National Laboratory }\end{array}$ \\
\hline LBNL & Masanet & Eric & \\
\hline LBNL & Nordman & Bruce & \\
\hline LBNL & Sartor & Dale & \\
\hline LBNL & Tschudi & Bill & Program Manager \\
\hline Lehman Brothers & Salmon & Rick & Vice President \\
\hline Liebert & MacCleary & Randy & VP and GM Liebert Power business \\
\hline Liebert & Madara & Steve & $\begin{array}{l}\text { VP and GM Liebert Environmental } \\
\text { business (cooling/air conditioning) }\end{array}$ \\
\hline Liebert & Panfil & Peter & VP Engineering Liebert Power \\
\hline Liebert & Pouchet & Jack & Director Marquee Accounts \\
\hline LSI Logic Corporation & Gee & Linda & Product Environmental Compliance \\
\hline LV Power Ltd & Applebaum & Aaron & General Manager \\
\hline Michaud Cooley Erickson & Herr & Guy C. & Vice President \\
\hline
\end{tabular}




\begin{tabular}{|c|c|c|c|}
\hline Microsoft & Gauthier & David & $\begin{array}{l}\text { Project Manager, Datacenter } \\
\text { Development }\end{array}$ \\
\hline Microsoft & O'Reilly & Jeff & \\
\hline Microsoft & Souarez & Amaya & $\begin{array}{l}\text { Manager, Capacity Planning \& } \\
\text { Standards } \\
\text { GFS Datacenter Services }\end{array}$ \\
\hline $\begin{array}{l}\text { Micro-Tech Consultants } \\
\text { (MTC) }\end{array}$ & Mankikar & Mohan & President \\
\hline Modius & Compiano & Craig & CEO \\
\hline Modius & Yeack & Bill & CFO \\
\hline $\begin{array}{l}\text { Morrison Hershfield } \\
\text { Corporation }\end{array}$ & Richard & John & $\begin{array}{l}\text { Director - Business DevelopmentData } \\
\text { Center Engineering }\end{array}$ \\
\hline $\begin{array}{l}\text { Natural Resources } \\
\text { Defense Council }\end{array}$ & Horowitz & Noah & Sr. Scientist \\
\hline On Semiconductor & Jandhyala & Sri & AC-DC Strategic Marketing Manager \\
\hline On Semiconductor & Mullett & Chuck & Principle Systems Engineer \\
\hline Oracle USA & Khattar & Mueksh & Energy Director \\
\hline Oracle USA & Shuder & James & Snr. Director Development \\
\hline $\begin{array}{l}\text { Pacific Gas and Electric } \\
\text { Company }\end{array}$ & Bramfitt & Mark J & High Tech Segment Lead \\
\hline $\begin{array}{l}\text { Pacific Gas and Electric } \\
\text { Company }\end{array}$ & Dunckel & William C & Sr. Project Manager \\
\hline $\begin{array}{l}\text { Pacific Gas and Electric } \\
\text { Company }\end{array}$ & Rongere & Francois & \\
\hline $\begin{array}{l}\text { Pacific Gas and Electric } \\
\text { Company }\end{array}$ & Villa & Tony & MS Server Product Manager \\
\hline Qimonda North America & Barth & Roland & \\
\hline Sabey DataCenter & Sasser & John & Operations Manager \\
\hline SGI, Inc. & McCann & Tim & Chief Engineer \\
\hline SGI, Inc. & Varney & R. Victor & $\begin{array}{l}\text { VP of Marketing \& Product Business } \\
\text { Management }\end{array}$ \\
\hline $\begin{array}{l}\text { Silicon Valley Leadership } \\
\text { Group }\end{array}$ & Pfeifer & Ray & Director - Data Center Demo Project \\
\hline Smart Works, Inc. & Diesso & Dan & \\
\hline SPEC & Lange & Klaus-Dieter & SPECpower Subcommittee Chair \\
\hline Spraycool & Tanner & Brandon & Director, Business Development \\
\hline Sun Microsystems & Bapat & Subodh & \\
\hline Sun Microsystems & Greenhill & David & Chief Engineer Niagara Systems \\
\hline Sun Microsystems & Symanski & Dennis & Worldwide Compliance Officer \\
\hline
\end{tabular}




\begin{tabular}{|l|l|l|l|}
$\begin{array}{l}\text { Super Micro Computer, } \\
\text { Inc. }\end{array}$ & Kalodrich & Michael & \\
\hline Teneros, Inc. & Asmersom & Kahsai & Senior NPI Program Manager \\
\hline Terranovum & Bolioli & Tom & \\
\hline Terrapin Systems & Becerra & Chris & \\
\hline UC Berkeley & Armbrust & Michael & \\
\hline UC Berkeley & Bodik & Peter & \\
\hline UC Berkeley & Shehabi & Arman & \\
\hline UC Berkeley & Stanley & John & \\
\hline Uptime Institute & Brill & Kenneth & Executive Director \\
\hline Uptime Institute & Sullivan & Robert & Senior Consultant \\
\hline VERDIEM & Twito & Bruce & CTO, V.P. Product Development \\
\hline Via Technologies & Wang & Cedric & \\
\hline Via Technologies & Wang & Johnny & \\
\hline Virginia Tech & Cameron & Kirk W. & \\
\hline VMWare & Balkansky & Bogomil & Director, Product Marketing \\
\hline VMWare & Gupta & Alok & Director, R\&D \\
\hline VMWare & Rob & Smoot & Product Marketing \\
\hline VMWare & Wilkerson & Mike & Manager, Systems Engineering \\
\hline $\begin{array}{l}\text { Weber Shandwick } \\
\text { Worldwide }\end{array}$ & Reddy & Dave & Vice President \\
\hline Wells Fargo Bank & Gedney & Cathie & Regional Facilities Mgr, AVP \& FMA \\
\hline Ziff Davis Media & Primesberger & Chris J. & Senior Writer, eWeek \\
\hline
\end{tabular}




\section{Appendix 3. Fuel Cell Installations in Data Centers and Related Premium Power Applications}

\section{Table A3-1. Fuel Cell Installations in Data Centers and Related Premium Power Applications}

\begin{tabular}{|c|c|c|c|c|c|c|c|}
\hline Site & Year & $\begin{array}{c}\text { Capacity } \\
\text { KW }\end{array}$ & Manufacturer & City & State & DG Type & Comments \\
\hline NYSERDA headquarters & 2006 & $2 \times 5$ & Plug Power & Albany & NY & $\begin{array}{l}\text { Prime } \\
\text { Power }\end{array}$ & $\begin{array}{l}\text { Two fuel cells and a photovoltaic (PV) awning system provide } \\
\text { power to the headquarters' systems including computer, } \\
\text { security and phones. The solar electric awning will power one- } \\
\text { half of NYSERDA's computer-driven power-load while } \\
\text { inverters will convert 3.6 kilowatts of direct current produced } \\
\text { by the solar modules, into alternating current. }\end{array}$ \\
\hline The Stella Group Ltd. & 2005 & $1 \times 5$ & Plug Power & Arlington & VA & Back-up & $\begin{array}{l}\text { The fuel cell is dedicated to certain circuits within the office } \\
\text { building, providing back-up power and power quality for the } \\
\text { circuits serving the lighting, computers and office machines } \\
\text { (telephone system, security system, fax and copier). It can } \\
\text { also be directed to charge the battery banks in both the office } \\
\text { building and the adjacent solar home of the Stella Group's } \\
\text { founder. }\end{array}$ \\
\hline Guaranty Savings Building & 2004 & $3 \times 200$ & UTC Power & Fresno & $\mathrm{CA}$ & $\mathrm{CHP}$ & $\begin{array}{l}\text { The building is a } 12 \text { story, } 100,000 \text { square foot, office tower, } \\
\text { which will house the INS Division of the Homeland Security } \\
\text { Department and the Tax Payer Advocacy Division of the IRS. } \\
\text { The fuel cells running on natural gas operate in grid parallel } \\
\text { configuration. The project includes a Multi Unit Load Sharing } \\
\text { (MULS) System and static switch that enables the fuel cells } \\
\text { to provide } 24 / 7 \text { power availability to the buildings mission } \\
\text { critical loads. The fuel cells include a UPS for the computer } \\
\text { server rooms on each floor, the communications systems, } \\
\text { building security systems, emergency lighting, elevator } \\
\text { motors, and stairwell ventilation fans. } \\
\text { The fuel cells provide two categories of waste heat. They } \\
\text { provide } 1,400,000 \text { Btu/hr at } 250^{\circ} F \text { high grade heat, and } \\
1,400,000 \text { Btu/hr at } 150^{\circ} F \text { low grade heat. The high grade } \\
\text { heat is piped to a } 120 \text { ton adsorption chiller to supply a } \\
\text { cooling load to the bottom three floors of the building. The } \\
\text { return side of the chiller thermal supply loop supplements the } \\
\text { buildings domestic hot water supply. The low grade heat is } \\
\text { piped to the heating coils associated with water source heat } \\
\text { pumps that have been installed throughout the building to } \\
\text { provide space heating for offices, hallways and ground floor } \\
\text { common areas. }\end{array}$ \\
\hline Naval Oceanic Center & 1997 & $1 \times 200$ & UTC Power & $\begin{array}{l}\text { Stennis } \\
\text { Space } \\
\text { Center }\end{array}$ & MS & $\mathrm{CHP}$ & $\begin{array}{l}\text { The fuel cell is located at the NAVOCEANO Computer } \\
\text { Programming Operations Center (building 1003) which } \\
\text { houses a computer center, library and laboratory. The fuel } \\
\text { cell thermal output heats hot water used in the air handlers } \\
\text { for space heating and for reheating cooled air to control } \\
\text { humidity. (Decommissioned 2002) }\end{array}$ \\
\hline Ramapo College & 2000 & $2 \times 200$ & UTC Power & Mahwah & $\mathrm{NJ}$ & $\mathrm{CHP}$ & $\begin{array}{l}\text { Grid parallel. Supplies power and thermal energy (hot water, } \\
\text { space heating) to a student dormitory and a core academic } \\
\text { building complex (housing a computer center, telephone } \\
\text { exchange and cable TV station). }\end{array}$ \\
\hline $\begin{array}{l}\text { U.S. Merchant Marine } \\
\text { Academy }\end{array}$ & 2002 & $3 \times 5$ & Plug Power & Kings Point & NY & Back-up & one year demonstration of new backup/UPS product \\
\hline $\begin{array}{l}\text { Gabreski Air National } \\
\text { Guard, base telephone } \\
\text { exchange }\end{array}$ & 2004 & $4 \times 1$ & ReliOn & $\begin{array}{l}\text { Westhamp- } \\
\text { ton }\end{array}$ & NY & Back-up & $\begin{array}{l}\text { The fuel cells are connected to the } 48 \mathrm{~V} \text { battery string on a } \\
\text { new uninterruptible power supply (UPS) system installed for } \\
\text { this project. One year demonstration }\end{array}$ \\
\hline $\begin{array}{l}\text { Patuxent Naval Air Station } \\
\text { office building }\end{array}$ & 2004 & $1 \times 5$ & Plug Power & $\begin{array}{l}\text { Patuxent } \\
\text { River }\end{array}$ & MD & $\mathrm{CHP}$ & $\begin{array}{l}\text { Powered } 9 \text { desktop computers, office lighting, oil furnace, } \\
\text { and life support systems for animals on display in } \\
\text { environmental / conservation building. Grid connected. } \\
\text { Excess power transferred to the grid. Cogenerated heat used } \\
\text { to provide heat to the building during cold months. (1-year } \\
\text { DoD demonstration) }\end{array}$ \\
\hline
\end{tabular}


Table A3-1: Fuel Cell Installations in Data Centers and Related Premium Power Applications (continued)

\begin{tabular}{|c|c|c|c|c|c|c|c|}
\hline Site & Year & $\begin{array}{c}\text { Capacity } \\
\text { KW }\end{array}$ & Manufacturer & City & State & DG Type & Comments \\
\hline $\begin{array}{l}\text { Fort Gordon Army } \\
\text { University of Technology } \\
\text { Resource Center }\end{array}$ & 2004 & $1 \times 5$ & Plug Power & $\begin{array}{l}\text { Fort } \\
\text { Gordon }\end{array}$ & GA & Back-up & $\begin{array}{l}\text { Provided back up to the servers that support the online virtual } \\
\text { training center. US DoD Residential PEM Fuel Cell } \\
\text { Demonstration Program FY } 2002 \text {. }\end{array}$ \\
\hline $\begin{array}{l}\text { Camp Roberts Army } \\
\text { National Guard Base }\end{array}$ & 2005 & $1 \times 200$ & UTC Power & $\begin{array}{l}\text { Paso } \\
\text { Robles }\end{array}$ & $\mathrm{CA}$ & $\begin{array}{l}\text { Prime } \\
\text { Power to } \\
\text { CHP }\end{array}$ & $\begin{array}{l}\text { The facility is the main US Army communications facility on } \\
\text { the West coast that provides worldwide communications } \\
\text { between the US National Command Authority and deployed } \\
\text { military units. } \\
\text { The fuel cell running on natural gas operates in grid parallel } \\
\text { configuration to provide power stabilization and reduce the } \\
\text { facility's electric demand from the grid. If the project receives } \\
\text { additional funding from a submitted proposal to the California } \\
\text { Self Generation Grant program the project will be expanded } \\
\text { to include a grid independent back-up generation component } \\
\text { and co-generation capabilities. The co-generation aspect, if } \\
\text { implemented, will capture the thermal energy to be used in } \\
\text { conjunction with an absorption chiller to assist with the } \\
\text { cooling loads of the data center. The fuel cell is equipped } \\
\text { with the high grade heat option which will provide } 400,000 \\
\text { Btu/hr of } 250 \text { oF heat at its high grade heat exchanger. This } \\
\text { will allow the fuel cell to support the thermal demand of an } \\
\text { absorption chiller having an output of approximately } 20-25 \\
\text { tons of cooling. }\end{array}$ \\
\hline Chevron Data Center & 2002 & $1 \times 200$ & UTC Power & $\begin{array}{c}\text { San } \\
\text { Ramon }\end{array}$ & $\mathrm{CA}$ & $\begin{array}{l}\text { Prime } \\
\text { Power }\end{array}$ & $\begin{array}{l}\text { Supports critical data and retail transaction systems. During a } \\
\text { power outage, special switching equipment ensures the fuel } \\
\text { cell will continue to provide electricity to these systems } \\
\text { without interruption. }\end{array}$ \\
\hline $\begin{array}{l}\text { Hamilton Sunstrand Data } \\
\text { Center }\end{array}$ & 1997 & $1 \times 200$ & UTC Power & $\begin{array}{l}\text { Windsor } \\
\text { Locks }\end{array}$ & $\mathrm{CT}$ & $\begin{array}{l}\text { Prime } \\
\text { Power }\end{array}$ & $\begin{array}{l}\text { This plant serves as the primary power source for the } \\
\text { Hamilton Sundstrand Data Center and Data Center UPS's. It } \\
\text { is considerred an ultra high reliable power source in that if an } \\
\text { outage occurs it is backed up by the grid via trnasfer switch } \\
\text { AND if the grid is unavailable, the load is transferred to a } 500 \\
\text { kw generator. }\end{array}$ \\
\hline First National Bank & 1999 & $4 \times 200$ & UTC Power & Omaha & $\mathrm{NE}$ & $\begin{array}{l}\text { Prime } \\
\text { Power }\end{array}$ & $\begin{array}{l}\text { Provides the main power for a critical data processing facility. } \\
\text { The bank is one of the largest credit card processors in the } \\
\text { nation. Independent verification of } 99.9999 \% \text { system } \\
\text { availability using Probabilistic Risk Analysis (PRA). }\end{array}$ \\
\hline $\begin{array}{l}\text { New York Power Authority, } \\
\text { State Office of General } \\
\text { Services - Suffolk Office }\end{array}$ & 2005 & $1 \times 200$ & UTC Power & Hauppage & NY & $\mathrm{CHP}$ & $\begin{array}{l}\text { The fuel cell is to supply power to the New York State } \\
\text { Regional Emergency Management Office, located in the } \\
\text { facility. The Regional Emergency Management Office } \\
\text { coordinates emergency planning and response for the New } \\
\text { York City and Long Island metropolitan areas. } \\
\text { The fuel cell running on natural gas is intended to operate in } \\
\text { grid parallel and grid independent modes. In the event of a } \\
\text { utility interruption, the fuel cell will isolate from the grid } \\
\text { parallel circuit and automatically reconnect to a backup circuit } \\
\text { within five seconds. Upon utility startup, the fuel cell will } \\
\text { automatically return to the grid parallel circuit. The thermal } \\
\text { energy from the fuel cell will be captured and used to } \\
\text { supplement the facility's heat and domestic hot water system. } \\
\text { The hot water loop will have a manual switch to allow for } \\
\text { connection to either the boiler return loop or the domestic hot } \\
\text { water loop depending on seasonal thermal demands. }\end{array}$ \\
\hline Verizon & 2005 & $7 \times 200$ & UTC Power & $\begin{array}{c}\text { Garden } \\
\text { City }\end{array}$ & $\mathrm{NY}$ & $\mathrm{CHP}$ & $\begin{array}{l}\text { Seven fuel cells generate power for a 292,000-square-foot } \\
\text { facility that provides telephone and data services to some } \\
35,000 \text { customers on Long Island. And it's connected to the } \\
\text { commercial power grid as backup. Waste heat is used for } \\
\text { heating and cooling the facility }\end{array}$ \\
\hline
\end{tabular}

Source: Energy and Environmental Analysis CHP Database (2006) 


\section{Case Studies of Combined Heat and Power Applications at Data Centers}

\section{Example Fuel Cell Application}

In April 2002, Verizon Communications was awarded a U.S. Department of Energy (DOE) and New York State Energy Research and Development Authority (NYSERDA) grant through programs aimed at supporting distributed energy resources in applications for data processing and telecommunications. As part of its “Central Office of the Future” project, Verizon installed multiple fuel cells and reciprocating engine generators to power a large central communications and data facility in New York (the Garden City project). The fuel cells were configured for CHP, utilizing waste heat from the fuel cells to provide thermal energy to the site as well. The project was designed to increase understanding of controls for multiple DG units and to utilize lowgrade heat for CHP benefits (CNET 2006).

Verizon's Garden City project is unique because it uses fuel cells as its primary source of energy. Seven fuel cells generate power for the 292,000-square-foot facility that provides telephone and data services to roughly 35,000 customers on Long Island. It is connected to the commercial power grid as backup (CNET 2006).

Verizon's benefits from the system are:

- $\$ 680,000$ per year in operating cost savings.

- Higher facility reliability.

- Displacement of one-third of its electric air conditioning load to thermally activated cooling.

- Lower emissions than those produced by central station power-11 million pounds per year less $\mathrm{CO}_{2}$ than would have been produced by a fossil-fueled central station power plant.

- Higher overall efficiency.

These benefits are mitigated somewhat by the current high cost of fuel cell power equipment. Verizon spent $\$ 13$ million on the facility-making the payback about 20 years without any type of incentives. Even with the incentives that Verizon received, the overall system costs, including capital recovery, are higher than for a conventional system (CNET 2006).

\section{Example Reciprocating Engine Application}

Network Appliance, Inc., an enterprise network storage provider, installed a state-of-the-art combined heat and power system for their facility in Sunnyvale, California (Engle 2005). Three $275 \mathrm{~kW}$ internal combustion engine packages use natural gas to produce electricity, which is fed to a UPS system that uses flywheels to provide short-term energy instead of batteries.

The waste heat from the engines is used to produce air conditioning for the data center using three 120 ton adsorption chillers. Adsorption chillers were used instead of the more common lithium bromide absorption chillers because the silica gel and water system that adsorption units are based on makes more effective use of the lower temperature heat available from the engine jacket water. 
The \$2.4 million system meets 80 percent of the facility’s electricity needs. The capital cost to Network Appliance was reduced by $\$ 800,000$ as a result of California's self generation incentive program (SGIP). Network Appliance estimates its $\$ 1.2$ million annual electricity bill for its research and development building will be cut by two-thirds. Company management first considered developing their own DG system during the rolling blackouts of 2002, as there was a strong concern that the company's mission critical power needs could not be adequately met without onsite power generation.

\section{Example Gas Turbine Application}

Qualcomm, a manufacturer and supplier of information technology and communications equipment, has made numerous energy saving investments at its office/data center world headquarters in San Diego, California. Some of these improvements have included lighting retrofits; HVAC upgrades; improvements to the building envelope; installation of a $500 \mathrm{~kW}$ solar photovoltaic system; use of hybrid vehicles for corporate shuttle service; and incorporation of efficient CHP to provide power, cooling, and hot water to the facility. These measures reduced energy demand by 10 million $\mathrm{kWh}$ per year and reduced $\mathrm{CO}_{2}$ emissions by 4,000 tons per year between 1993 and 2002.

Qualcomm installed a 1.6 MW gas turbine CHP system at their facility in the early 1990s. The system has saved more than $\$ 500,000$ annually in cooling costs from two 500-ton absorption chillers driven by heat recovered from the gas turbines. An additional $\$ 100,000$ is saved annually through a heat recovery unit that supplies hot water to the facility. Onsite electricity generation reduces demand for utility energy by over $14,000,000 \mathrm{kWh}$ per year, saving another $\$ 122,000$. Total annual savings achieved by the CHP system is more than $\$ 775,000$. Based on their positive experience with the original gas turbine system, Qualcomm is currently expanding their campus CHP system, installing two high-efficiency 4.8 MW recuperated gas turbines with heat recovery. One turbine will be dedicated to a new data center at the headquarters campus, supplying both power and cooling to the facility. 


\title{
Appendix 4: Scenario modeling approach and assumptions
}

\section{Introduction}

The general modeling approach described in Chapter 2 was employed to project future energy use for all scenarios analyzed in this report. In order to capture the efficiency trends necessary to model these scenarios (such as the effects of virtualization on the installed server base and the effects of power management use on the average server UEC), it was necessary to augment the modeling approach described in Chapter 2. The modeling details for each scenario are described in the remainder of this appendix.

The estimates described in this section were based on best available information and data at the time of this study. However, there are inherent uncertainties associated with the data and assumptions employed in this study, and therefore the estimates presented in this section should be interpreted as preliminary in nature. Recommendations for future work to reduce the uncertainties associated with these estimates are summarized in Chapter 8.

\section{Modeling Approach and Assumptions for the Historical Trends and Current Efficiency Trends Scenarios}

\author{
Estimation of U.S. Installed Server Base by Space Type
}

Current U.S. server market forecast data provided by market research firm IDC (IDC 2007a) were used to project the U.S. installed base of volume, mid-range, and high-end servers over the period 2007 to 2011 in all scenarios. Since the IDC data only contained forecasts to 2010 (reflected in Figure 2-3), the data were extended to 2011 by using the 2007 to 2010 compound annual growth rate (CAGR) for each server class as predicted by IDC. Given that the IDC projections were based on an extensive analysis of current U.S. market trends (including the growth of virtualization and its effects on the installed base of volume servers), these data were used as the U.S. installed server base projections in the current efficiency trends scenario.

Table A4-1 summarizes the projections for the U.S. installed server base by server class and space type for the current efficiency trends scenario. To derive the breakdown of server class by space type, the disaggregation approach previously described in Chapter 2 was used. ${ }^{1}$ The projections in Table A4-1 suggest that the U.S. volume server market will experience significant growth over the next five years, with the installed base rising by nearly $50 \%$ by 2011 . Conversely, the numbers of installed high-end and mid-range servers are expected to decline by $30 \%$ and $12 \%$, respectively, over the same time period.

\footnotetext{
${ }^{1}$ For volume servers in server closets over the period 2007 to 2011, a CAGR of 4\% was assumed based on recent server closet growth projections by Bailey et al. (2006). In each year from 2007 to 2011, the remaining non server closet volume servers were allocated to the remaining space types in a proportional manner based on the 2005 breakdown in Table 2-2.
} 
Table A4-1. Projected U.S. Installed Server Base (in 1000s) by Server Class and Space Type, Current Efficiency Trends Scenario, 2007 to 2011

\begin{tabular}{|r|c|c|c|c|c|}
\hline Volume servers in: & $\mathbf{2 0 0 7}$ & $\mathbf{2 0 0 8}$ & $\mathbf{2 0 0 9}$ & $\mathbf{2 0 1 0}$ & $\mathbf{2 0 1 1}$ \\
\hline Server closets & 1,870 & 1,945 & 2,023 & 2,104 & 2,188 \\
\hline Server rooms & 2,400 & 2,660 & 2,925 & 3,213 & 3,642 \\
\hline Localized data centers & 2,060 & 2,283 & 2,510 & 2,757 & 3,126 \\
\hline Mid-tier data centers & 1,860 & 2,062 & 2,267 & 2,490 & 2,823 \\
\hline Enterprise-class data centers & 3,639 & 4,033 & 4,435 & 4,871 & 5,522 \\
\hline Total volume & 11,829 & 12,982 & 14,160 & 15,434 & 17,300 \\
\hline Mid-range servers in: & $\mathbf{2 0 0 7}$ & $\mathbf{2 0 0 8}$ & $\mathbf{2 0 0 9}$ & $\mathbf{2 0 1 0}$ & $\mathbf{2 0 1 1}$ \\
\hline Server closets & 0 & 0 & 0 & 0 & 0 \\
\hline Server rooms & 17 & 17 & 16 & 16 & 15 \\
\hline Localized data centers & 55 & 53 & 52 & 52 & 48 \\
\hline Mid-tier data centers & 49 & 48 & 47 & 46 & 43 \\
\hline Enterprise-class data centers & 226 & 219 & 214 & 212 & 198 \\
\hline Total mid-range & 347 & 336 & 330 & 326 & 304 \\
\hline High-end servers in: & $\mathbf{2 0 0 7}$ & $\mathbf{2 0 0 8}$ & $\mathbf{2 0 0 9}$ & $\mathbf{2 0 1 0}$ & $\mathbf{2 0 1 1}$ \\
\hline Server closets & 0 & 0 & 0 & 0 & 0 \\
\hline Server rooms & 0 & 0 & 0 & 0 & 0 \\
\hline Localized data centers & 3 & 3 & 2 & 2 & 2 \\
\hline Mid-tier data centers & 3 & 2 & 2 & 2 & 2 \\
\hline Enterprise-class data centers & 13 & 12 & 11 & 11 & 10 \\
\hline Total high-end & 18 & 17 & 16 & 15 & 15 \\
\hline
\end{tabular}

Table A4-2 summarizes the projections for the U.S. installed base of volume servers by space type for the historical trends scenario, which assumes that no virtualization will occur among volume servers over the period 2007 to 2011. Because the server virtualization trends considered in this report are applicable only to volume servers, it was assumed that the projections for the U.S. installed base of high-end and mid-range servers summarized in Table A4-1 were also valid for the historical trends scenario (and all other scenarios considered in this report).

Table A4-2. Projected U.S. Installed Base of Volume Servers (in 1000s) by Space Type, Historical Trends Scenario, 2007 to 2011

\begin{tabular}{|c|c|c|c|c|c|}
\hline Volume servers in: & $\mathbf{2 0 0 7}$ & $\mathbf{2 0 0 8}$ & $\mathbf{2 0 0 9}$ & $\mathbf{2 0 1 0}$ & $\mathbf{2 0 1 1}$ \\
\hline Server closets & 1,873 & 1,971 & 2,079 & 2,190 & 2,271 \\
\hline Server rooms & 2,408 & 2,731 & 3,088 & 3,475 & 3,918 \\
\hline Localized data centers & 2,067 & 2,351 & 2,665 & 3,005 & 3,385 \\
\hline Mid-tier data centers & 1,867 & 2,123 & 2,407 & 2,714 & 3,057 \\
\hline Enterprise-class data centers & 3,652 & 4,154 & 4,709 & 5,310 & 5,980 \\
\hline Total volume & 11,866 & 13,330 & 14,949 & 16,693 & 18,611 \\
\hline
\end{tabular}

To derive the data in Table A4-2, it was first assumed that the virtualization trends included in the volume server installed base projections in Table A4- 1 were applicable to only $50 \%$ of the volume servers located in server closets. This assumption was based on the expectation that 
many server closets will only host one local workgroup server and are thus not candidates for virtualization.

Next, IDC (2007b) adjusted market forecast data were used to estimate the number of non server closet volume servers that would be eliminated from the installed base via current market trends toward virtualization. IDC predicted that worldwide volume server shipments that were once projected to increase $61 \%$ by 2010 are facing just 39\% growth during that same period due to increased virtualization. The above change in shipments was used, coupled with projections on volume server shipments and retirements in the IDC (2007a) dataset, to estimate the number of volume servers eliminated from the installed base each year due to virtualization. Finally, the eliminated servers each year were added back by space type in a proportional manner to arrive at the historical trends projections for volume servers in Table A4-2. .

To characterize the effects of volume server reduction via virtualization on the installed base in the scenario analyses, the physical server reduction (PSRR) ratio in year $i$ and space type $j$ was defined as follows:

$$
\begin{aligned}
& \left.\operatorname{PSRR}_{i j}=\text { (historical trends installed base of volume servers }\right)_{i j} / \\
& (\text { post-virtualization installed base of volume servers })_{i j}
\end{aligned}
$$

The definition of the above variable allowed the installed base of volume servers in a given year to be derived by coupling an assumed PSRR with the historical trends installed base projections in Table A4-2. Using Equation A4-1, it was estimated from Tables A4-1 and A4-2 that current trends toward volume server virtualization will lead to a PSRR of roughly 1.04 by the year 2011 for volume servers in server closets, and a PSRR of roughly 1.08 by the year 2011 for volume servers in all other space types.

\section{Estimation of Average Energy Use per Server}

Table A4-3 lists the projections of average UEC by server class for the historical trends scenario. These projections were estimated by extrapolating the 2000 to 2006 UEC trends in Table 2-4 out to 2011, using CAGR values derived from Koomey (2007). The CAGR for volume servers in Table A4-3 acknowledges the fact that the Koomey (2007) predicted a steady decrease in the growth rate of power use per server for volume servers over its five-year analysis period.

\section{Table A4-3. Projected Average UEC (in kWh/year) by Server Class, Historical Trends Scenario, 2007 to 2011}

\begin{tabular}{|l|c|c|c|c|c|c|}
\hline Server class & $\mathbf{2 0 0 7}$ & $\mathbf{2 0 0 8}$ & $\mathbf{2 0 0 9}$ & $\mathbf{2 0 1 0}$ & $\mathbf{2 0 1 1}$ & $\begin{array}{c}\mathbf{2 0 0 7 - 2 0 1 1} \\
\text { CAGR }\end{array}$ \\
\hline Volume & 2,017 & 2,068 & 2,106 & 2,147 & 2,186 & $2 \%$ \\
\hline Mid-range & 6,394 & 6,929 & 7,468 & 8,070 & 8,722 & $8 \%$ \\
\hline High-end & 76,295 & 81,624 & 86,849 & 92,662 & 98,864 & $7 \%$ \\
\hline
\end{tabular}

To project the average UEC by server class for the current efficiency trends scenario, there were several important issues to consider: (1) the increasing penetration of "energy efficient" volume servers in the installed base each year, (2) that virtualization would lead to an increase in the 
average processor utilization level for volume servers, and (3) the use of power management on applicable servers. These issues are discussed in more detail below.

The increasing penetration of "energy efficient" volume server models will tend to decrease the average UEC across all volume servers in the U.S. installed server base. First, the penetrations of “energy efficient” models by year and space type were estimated, as summarized in Table A4-4.

\section{Table A4-4. Percent of Installed Base of Volume Servers that is "Energy Efficient" by Space Type, Current Efficiency Trends Scenario, 2007 to 2011}

\begin{tabular}{|l|c|c|c|c|c|}
\hline \multirow{2}{*}{ Volume servers in: } & \multicolumn{5}{|c|}{$\%$ of installed volume server base that is energy } \\
& \multicolumn{5}{|c|}{ efficient } \\
\cline { 2 - 6 } & $\mathbf{2 0 0 7}$ & $\mathbf{2 0 0 8}$ & $\mathbf{2 0 0 9}$ & $\mathbf{2 0 1 0}$ & $\mathbf{2 0 1 1}$ \\
\hline Server closets & $1 \%$ & $3 \%$ & $5 \%$ & $8 \%$ & $12 \%$ \\
\hline Server rooms & $1 \%$ & $3 \%$ & $6 \%$ & $9 \%$ & $12 \%$ \\
\hline Localized data centers & $1 \%$ & $3 \%$ & $6 \%$ & $9 \%$ & $12 \%$ \\
\hline Mid-tier data centers & $1 \%$ & $3 \%$ & $6 \%$ & $9 \%$ & $12 \%$ \\
\hline Enterprise-class data centers & $1 \%$ & $3 \%$ & $6 \%$ & $9 \%$ & $12 \%$ \\
\hline
\end{tabular}

The estimates in Table A4-4 were generated using a server stock turnover accounting approach based on projected volume server shipments and retirements by year from IDC (2007a) for the current efficiency trends scenario. Because the number of installed servers in server closets was expected to grow more slowly than the number of installed servers in server rooms and data centers, the server closet penetration of "energy efficient" servers is slightly different than those of the other space types.

Using the data in Table A4-4, the weighted average volume server UEC ( $\left.U E C_{A V G}\right)$ in year $i$ for space type $j$ was calculated using the following relation:

$$
\left(U E C_{A V G}\right)_{i j}=\left(U E C_{H T}\right)_{i j} *\left(1-x_{i j}+x_{i j} y_{i j}\right)
$$

where for each year $i$ and space type $j, U E C_{H T}$ is the average historical trends volume server UEC from Table A4-3, $x$ is the percentage of the installed volume server base that is "energy efficient" from Table A4-4, and $y$ is the \% savings in UEC associated with an "energy efficient" volume server as compared to the historical trends volume server. (Recall from Chapter 3 that the assumed value of $y$ in this report is $25 \%$ ).

To account for the energy effects of increased processor utilization due to virtualization, as well as the energy effects of power management, representative industry data showing the relationship between system (i.e., server) energy use, processor utilization, and power management state (i.e., on or off) were used in this report. The data used to characterize this relationship are summarized in Figures A4-1a (AMD 2006) and A4-1b (Nordman 2005). Figure A4-1a depicts this relationship for servers manufactured in years 2006 and later; Figure A4-1b depicts this relationship for servers manufactured in years 2005 and earlier. The use of two separate graphs acknowledges the shift in the relationship between server energy use, processor utilization, and power management state that has occurred over time. The manufacturing age of 
servers comprising the installed base each year was derived using the stock turnover approach described previously.

For all volume servers, an average processor utilization level of $10 \%$ was assumed in the absence of virtualization based on the analysis of server energy efficiency trends presented in Chapter 3.

For all volume servers that are subject to virtualization, the average volume server processor utilization level after server reduction $\left(U_{A F T E R}\right)$ in year $i$ by space type $j$ was calculated using the following relation:

$$
U_{A F T E R}=10 \% *\left(P S R R_{i j} *\left(1-z_{i j}\right)+z_{i j}\right)+w
$$

where $z_{i j}$ is equal to the percentage of servers eliminated during virtualization efforts in year $i$ in space type $j$ that are not replaced by virtual machines. The variable $z$ was included to account for the fact that in many server reduction efforts, it is possible to identify servers running legacy applications that are no longer needed (and therefore these servers do not need to be converted to virtual machines). The variable $w$ accounts for the software overhead associated with running virtualization software on the host machine, and ranged from $0 \%$ to $5 \%$ depending on the percentage of installed volume server base that served as host servers.

Figure A4-1a. Assumed Relationship between Power Management, Processor Utilization, and System Power for Servers Manufactured in 2006 and Years Later

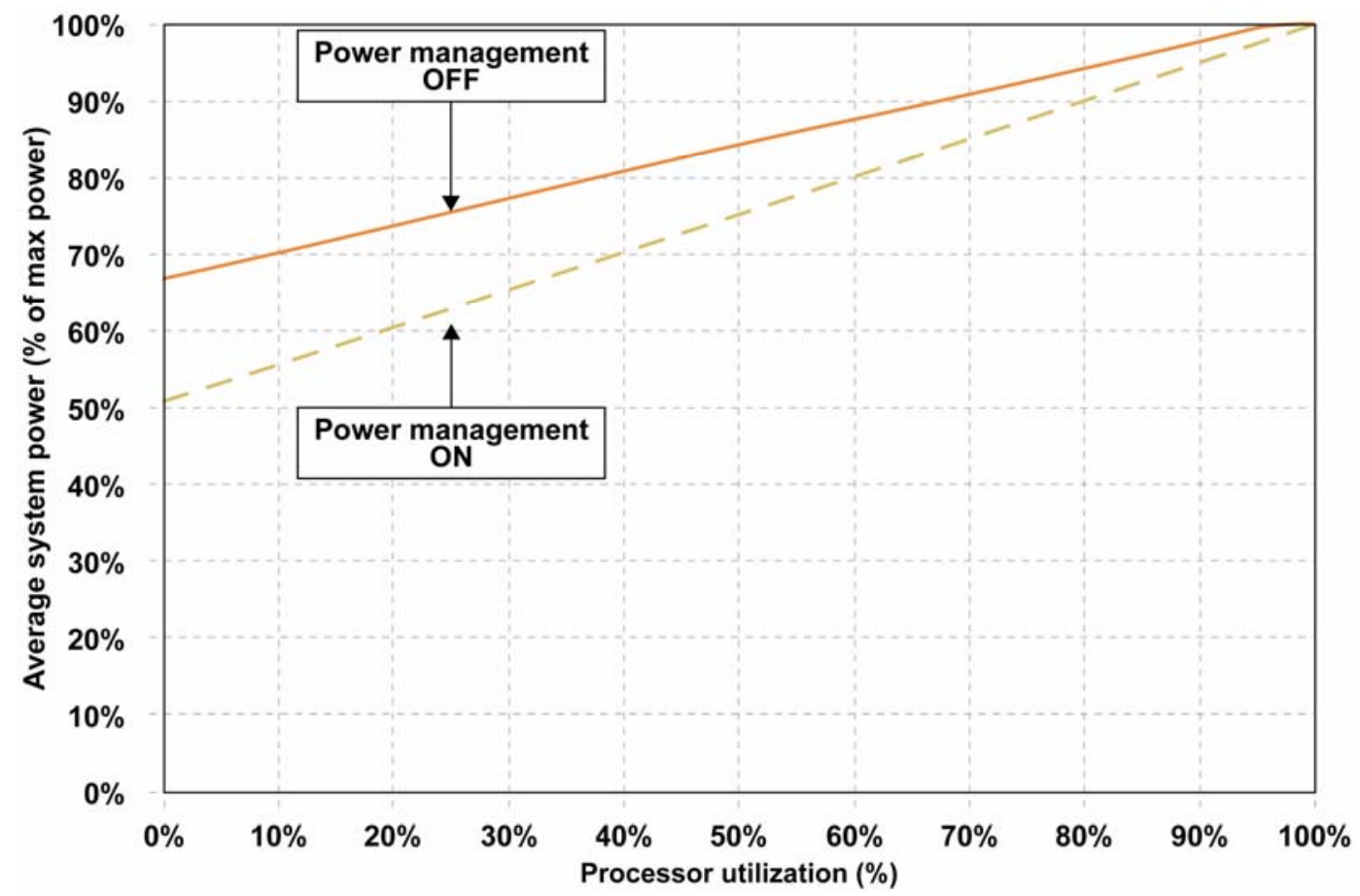

Source: Derived from AMD (2006) 
Figure A4-1b. Assumed Relationship between Power Management, Processor Utilization, and System Power for Servers Manufactured in 2005 and Years Prior

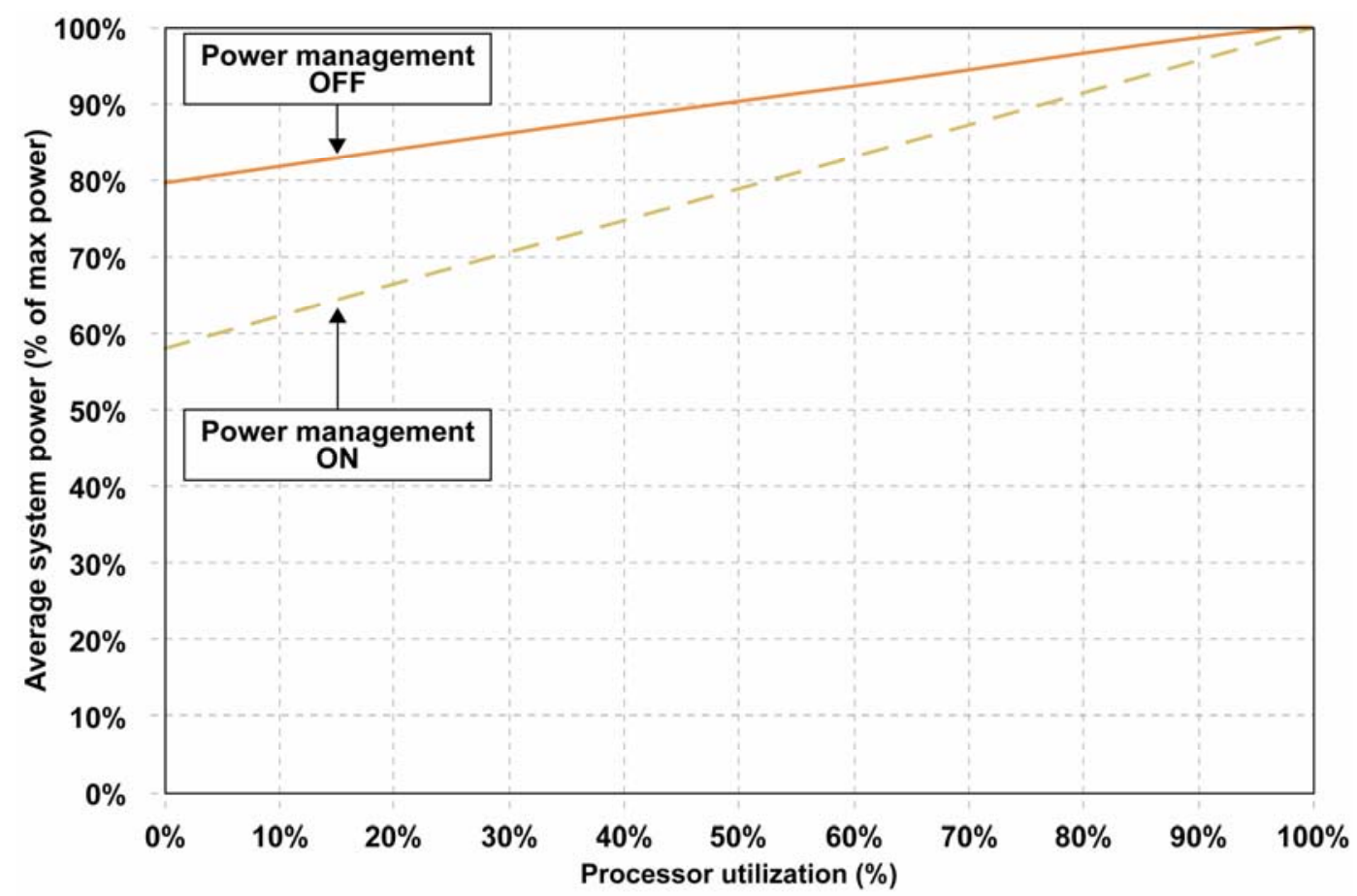

Source: Derived from Nordman (2005)

Using Equations A4-2 and A4-3 and the relationships depicted in Figures A4-1a and A4-1b, it was possible to derive estimates for the average volume server UEC by year and space type based on scenario assumptions for: (1) the annual penetration of "energy efficient" volume servers, (2) the PSRR due to virtualization, and (3) the average level of power management utilization across the installed volume server base.

For mid-range and high-end servers, it was assumed that the average UECs for the historical trends scenario (in Table A4-3) would also be valid for the current efficiency trends scenario (and all other scenarios considered in this report), since the observed trends toward more efficient servers are occurring in the volume server market.

It was further assumed that power management would be applicable to the mid-range server class. To calculate the average UEC for mid-range servers under the power management assumptions of each scenario, an average processor utilization level of $20 \%$ was assumed based on estimates compiled from industry experts (Dietrich 2007; U.S. EPA 2007). Based on this assumption, the data in Figures A4-1a and A4-1b were used to estimate the average energy savings associated with power management.

Table A4-5 summarizes the estimates for the average volume server UEC by space type for the current efficiency trends scenario over the period 2007 to 2011, based on the methods described above. Table A4-6 summarizes the estimates of average UEC for mid-range and high-end 
servers (which don't vary by space type) for the current efficiency trends scenario over the same time period.

Table A4-5. Projected Average Volume Server UEC (in kWh/year) by Space Type, Current Efficiency Trends Scenario, 2007 to 2011

\begin{tabular}{|l|c|c|c|c|c|}
\hline Volume servers in: & $\mathbf{2 0 0 7}$ & $\mathbf{2 0 0 8}$ & $\mathbf{2 0 0 9}$ & $\mathbf{2 0 1 0}$ & $\mathbf{2 0 1 1}$ \\
\hline Server closets & 1,959 & 2,006 & 2,035 & 2,059 & 2,079 \\
\hline Server rooms & 1,960 & 2,004 & 2,033 & 2,058 & 2,080 \\
\hline Localized data centers & 1,960 & 2,004 & 2,033 & 2,058 & 2,080 \\
\hline Mid-tier data centers & 1,960 & 2,004 & 2,033 & 2,058 & 2,080 \\
\hline Enterprise-class data centers & 1,960 & 2,004 & 2,033 & 2,058 & 2,080 \\
\hline
\end{tabular}

Table A4-6. Projected Average UEC (in kWh/year) for Mid-range and High-end Servers, Current Efficiency Trends Scenario, 2007 to 2011

\begin{tabular}{|l|c|c|c|c|c|}
\hline Server class & $\mathbf{2 0 0 7}$ & $\mathbf{2 0 0 8}$ & $\mathbf{2 0 0 9}$ & $\mathbf{2 0 1 0}$ & $\mathbf{2 0 1 1}$ \\
\hline Mid-range & 6,254 & 6,791 & 7,333 & 7,928 & 8,568 \\
\hline High-end & 76,295 & 81,624 & 86,849 & 92,662 & 98,864 \\
\hline
\end{tabular}

Estimation of Total Energy Use for U.S. Servers by Space Type

Table A4-7 summarizes the total projected energy use of U.S. servers by space type for the historical trends and current efficiency trends scenarios, based on the installed server base and average UEC assumptions described above. 
Table A4-7. Projected Total Energy Use of U.S. Servers (in billion kWh/year) by Space Type, Historical Trends and Current Efficiency Trends Scenarios, 2007 to 2011

\begin{tabular}{|c|c|c|c|c|c|}
\hline \multicolumn{6}{|c|}{ Historical trends scenario } \\
\hline Space type & 2007 & 2008 & 2009 & 2010 & 2011 \\
\hline Server closet & 3.8 & 4.1 & 4.4 & 4.7 & 5.0 \\
\hline Server room & 5.0 & 5.8 & 6.6 & 7.6 & 8.7 \\
\hline Localized data center & 4.7 & 5.4 & 6.2 & 7.1 & 8.0 \\
\hline Mid-tier data center & 4.3 & 4.9 & 5.6 & 6.4 & 7.3 \\
\hline Enterprise-class data center & 9.8 & 11.1 & 12.5 & 14.1 & 15.8 \\
\hline Total & 27.6 & 31.3 & 35.3 & 39.9 & 44.8 \\
\hline \multicolumn{6}{|c|}{ Current efficiency trends scenario } \\
\hline Space type & 2007 & 2008 & 2009 & 2010 & 2011 \\
\hline Server closet & 3.7 & 3.9 & 4.1 & 4.3 & 4.5 \\
\hline Server room & 4.8 & 5.4 & 6.1 & 6.7 & 7.7 \\
\hline Localized data center & 4.6 & 5.2 & 5.7 & 6.3 & 7.1 \\
\hline Mid-tier data center & 4.1 & 4.6 & 5.1 & 5.7 & 6.4 \\
\hline Enterprise-class data center & 9.5 & 10.5 & 11.6 & 12.7 & 14.2 \\
\hline Total & 26.8 & 29.7 & 32.6 & 35.8 & 40.0 \\
\hline
\end{tabular}

Estimation of Energy Use for Storage Devices and Network Equipment

To project the energy use associated with enterprise storage devices, forecast data on the energy use of enterprise HDD storage devices was employed (Osterberg 2007). As described in the efficiency trends section of Chapter 3, an energy efficiency improvement of $7 \%$ over the period 2007 to 2011 was assumed for the current efficiency trends scenario. For the historical trends scenario, it was assumed that an average power use of 14 watts per drive in 2006 would remain constant over the period 2007 to 2006 (Osterberg 2007). As in Chapter 2, 100\% was added to these energy use estimates to account for storage control, power supply losses, and other storage system components. Total storage energy use was then allocated to localized, mid-tier, and enterprise-class data centers proportionally based on the number of installed servers in those space types. The projections for the total annual energy consumed by enterprise storage devices for the historical trends and current efficiency trends scenarios are summarized in Table A4-8. 
Table A4-8. Projected Total Energy Use of U.S. Enterprise Storage Devices (in billion kWh/year) by Space Type, Historical Trends and Current Efficiency Trends Scenarios, 2007 to 2011

\begin{tabular}{|c|c|c|c|c|c|}
\hline \multicolumn{6}{|c|}{ Historical trends scenario } \\
\hline Space type & 2007 & 2008 & 2009 & 2010 & 2011 \\
\hline Server closet & 0 & 0 & 0 & 0 & 0 \\
\hline Server room & 0 & 0 & 0 & 0 & 0 \\
\hline Localized data center & 1.08 & 1.44 & 1.86 & 2.50 & 3.02 \\
\hline Mid-tier data center & 0.97 & 1.30 & 1.68 & 2.26 & 2.73 \\
\hline Enterprise-class data center & 1.97 & 2.63 & 3.37 & 4.52 & 5.45 \\
\hline Total & 4.02 & 5.37 & 6.90 & 9.27 & 11.20 \\
\hline \multicolumn{6}{|c|}{ Current efficiency trends scenario } \\
\hline Space type & 2007 & 2008 & 2009 & 2010 & 2011 \\
\hline Server closet & 0 & 0 & 0 & 0 & 0 \\
\hline Server room & 0 & 0 & 0 & 0 & 0 \\
\hline Localized data center & 1.04 & 1.38 & 1.77 & 2.29 & 2.76 \\
\hline Mid-tier data center & 0.94 & 1.24 & 1.59 & 2.07 & 2.49 \\
\hline Enterprise-class data center & 1.90 & 2.51 & 3.21 & 4.15 & 4.98 \\
\hline Total & 3.88 & 5.14 & 6.57 & 8.51 & 10.24 \\
\hline
\end{tabular}

To project the total energy use of network equipment by space type for the historical trends scenario, the 2000 to 2006 network equipment energy use trends (in Table 2-7) were extrapolated out to 2011. Due to lack of data on the likely energy efficiency trends associated with network equipment over the next five years, it was assumed that the trends established in the historical trends scenario would be valid for all scenarios considered in this study. The projections for the energy use of network equipment by space type for the historical trends scenario are summarized in Table A4-9.

Table A4-9. Projected Total Energy Use of Network Equipment (in billion kWh/year) by Space Type, Historical Trends Scenario, 2007 to 2011

\begin{tabular}{|r|c|c|c|c|c|}
\hline Space type & $\mathbf{2 0 0 7}$ & $\mathbf{2 0 0 8}$ & $\mathbf{2 0 0 9}$ & $\mathbf{2 0 1 0}$ & $\mathbf{2 0 1 1}$ \\
\hline Server closet & 0.20 & 0.21 & 0.23 & 0.25 & 0.26 \\
\hline Server room & 0.67 & 0.80 & 0.95 & 1.13 & 1.31 \\
\hline Localized data center & 0.65 & 0.77 & 0.90 & 1.07 & 1.23 \\
\hline Mid-tier data center & 0.58 & 0.69 & 0.81 & 0.96 & 1.11 \\
\hline Enterprise-class data center & 1.31 & 1.52 & 1.76 & 2.07 & 2.36 \\
\hline Total & 3.41 & 3.99 & 4.64 & 5.47 & 6.28 \\
\hline
\end{tabular}

Estimation of Energy Used by Site Infrastructure Systems

For the historical trends scenario, it was assumed that the estimated 2000 to 2006 PUE ratio of 2.0 would remain frozen over the period 2007 to 2011. As discussed in Chapter 3, it was estimated in the current efficiency trends scenario that over the next five years that this 2.0 historical PUE ratio would drop to 1.9 in a linear fashion by 2011. It was assumed that this $5 \%$ drop will be valid for all space types in the current efficiency trends scenario. The resulting 
projections for the total energy use attributable to site infrastructure systems by space type for the historical trends and current efficiency trends scenarios are summarized in Table A4-10.

Table A4-10. Projected Total Energy Use of Infrastructure systems (in billion kWh/year) by Space Type, Historical Trends and Current Efficiency Trends Scenarios, 2007 to 2011

\begin{tabular}{|c|c|c|c|c|c|}
\hline \multicolumn{6}{|c|}{ Historical trends scenario } \\
\hline Space type & 2007 & 2008 & 2009 & 2010 & 2011 \\
\hline Server closet & 4.0 & 4.3 & 4.6 & 4.9 & 5.2 \\
\hline Server room & 5.6 & 6.6 & 7.6 & 8.7 & 10.0 \\
\hline Localized data center & 6.5 & 7.7 & 9.0 & 10.7 & 12.3 \\
\hline Mid-tier data center & 5.8 & 6.9 & 8.1 & 9.6 & 11.1 \\
\hline Enterprise-class data center & 13.1 & 15.2 & 17.6 & 20.7 & 23.6 \\
\hline Total & 35.0 & 40.6 & 46.9 & 54.6 & 62.3 \\
\hline \multicolumn{6}{|c|}{ Current efficiency trends scenario } \\
\hline Space type & 2007 & 2008 & 2009 & 2010 & 2011 \\
\hline Server closet & 3.8 & 4.0 & 4.1 & 4.2 & 4.3 \\
\hline Server room & 5.4 & 6.0 & 6.6 & 7.2 & 8.1 \\
\hline Localized data center & 6.2 & 7.0 & 7.9 & 8.9 & 10.0 \\
\hline Mid-tier data center & 5.6 & 6.3 & 7.1 & 8.0 & 9.0 \\
\hline Enterprise-class data center & 12.5 & 14.0 & 15.5 & 17.4 & 19.4 \\
\hline Total & 33.4 & 37.3 & 41.2 & 45.8 & 50.9 \\
\hline
\end{tabular}

\section{Modeling Approach and Assumptions for the Additional Efficiency Scenarios}

Estimation of U.S. Installed Server Base by Space Type

Table A4-11 summarizes the projections for the U.S. installed base of volume servers over the period 2007 to 2011 for each of the scenarios listed in Table 3-19. To derive the projections for each scenario, it was assumed that the PSRR each year would ramp up linearly to the ultimate 2011 PSRR listed in the assumptions for each scenario in Table 3-5.

Given that the improved operation scenario assumes the same 2011 PSRR as the current efficiency trends scenario, the projected installed base of volume servers remains unchanged between these two scenarios. However, in the best practice and state-of-the-art scenarios, the effects of increasingly aggressive server reductions through virtualization are quite obvious when observing the 2011 installed base for each scenario. 
Table A4-11. Projected U.S. Installed Base of Volume Servers (in 1000s) by Space Type, Alternative Scenarios, 2007 to 2011

\begin{tabular}{|c|c|c|c|c|c|}
\hline \multicolumn{6}{|c|}{ Improved operation scenario } \\
\hline Volume servers in: & 2007 & 2008 & 2009 & 2010 & 2011 \\
\hline Server closets & 1,870 & 1,945 & 2,023 & 2,104 & 2,188 \\
\hline Server rooms & 2,400 & 2,660 & 2,925 & 3,213 & 3,642 \\
\hline Localized data centers & 2,060 & 2,283 & 2,510 & 2,757 & 3,126 \\
\hline Mid-tier data centers & 1,860 & 2,062 & 2,267 & 2,490 & 2,823 \\
\hline Enterprise-class data centers & 3,639 & 4,033 & 4,435 & 4,871 & 5,522 \\
\hline Total volume & 11,829 & 12,982 & 14,160 & 15,434 & 17,300 \\
\hline \multicolumn{6}{|c|}{ Best practice scenario } \\
\hline Volume servers in: & 2007 & 2008 & 2009 & 2010 & 2011 \\
\hline Server closets & 1,767 & 1,760 & 1,762 & 1,766 & 1,707 \\
\hline Server rooms & 2,006 & 1,951 & 1,930 & 1,930 & 1,959 \\
\hline Localized data centers & 1,722 & 1,679 & 1,666 & 1,670 & 1,692 \\
\hline Mid-tier data centers & 1,556 & 1,517 & 1,505 & 1,508 & 1,529 \\
\hline Enterprise-class data centers & 3,043 & 2,967 & 2,943 & 2,950 & 2,990 \\
\hline Total volume & 10,095 & 9,873 & 9,806 & 9,823 & 9,878 \\
\hline \multicolumn{6}{|c|}{ State-of-the-art scenario } \\
\hline Volume servers in: & 2007 & 2008 & 2009 & 2010 & 2011 \\
\hline Server closets & 1,658 & 1,564 & 1,496 & 1,441 & 1,368 \\
\hline Server rooms & 1,783 & 1,438 & 1,123 & 869 & 784 \\
\hline Localized data centers & 1,531 & 1,237 & 969 & 751 & 677 \\
\hline Mid-tier data centers & 1,383 & 1,117 & 875 & 679 & 611 \\
\hline Enterprise-class data centers & 2,705 & 2,186 & 1,712 & 1,327 & 1,196 \\
\hline Total volume & 9,060 & 7,543 & 6,176 & 5,066 & 4,636 \\
\hline
\end{tabular}

\section{Estimation of Average Energy Use per Server}

The average volume server UECs by space type for each scenario were projected using the UEC calculation method explained in detail for the current efficiency trends scenario. This method took into account the following assumptions for each scenario listed in Table 3-5:

- the assumed annual penetration rate for "energy efficient" volume servers, based on stock turnover accounting

- the assumed 2011 PSRR due to virtualization,

- the estimated percentage of eliminated servers not replaced during virtualization efforts, and

- the estimated average level of power management utilization.

The resulting projections for average volume server UEC by space type over the period 2007 to 2011 are summarized in Table A4-12. When compared to the current efficiency trends scenario UEC projections (Table A4-5), the UEC projections for the improved operation scenario in Table A4-12 suggest that significant server-level energy savings can be achieved with the aggressive use of power management. 
Table A4-12. Projected Average Volume Server UEC (in kWh/year) by Space Type, Alternative Scenarios, 2007 to 2011

\begin{tabular}{|r|c|c|c|c|c|}
\hline \multicolumn{7}{|c|}{ Improved operation scenario } \\
\hline Volume servers in: & $\mathbf{2 0 0 7}$ & $\mathbf{2 0 0 8}$ & $\mathbf{2 0 0 9}$ & $\mathbf{2 0 1 0}$ & $\mathbf{2 0 1 1}$ \\
\hline Server closets & 1,505 & 1,580 & 1,643 & 1,673 & 1,689 \\
\hline Server rooms & 1,512 & 1,586 & 1,646 & 1,677 & 1,693 \\
\hline Localized data centers & 1,512 & 1,586 & 1,646 & 1,677 & 1,693 \\
\hline Mid-tier data centers & 1,512 & 1,586 & 1,646 & 1,677 & 1,693 \\
\hline Enterprise-class data centers & 1,512 & 1,586 & 1,646 & 1,677 & 1,693 \\
\hline \multicolumn{7}{|c|}{ Best practice scenario } \\
\hline Volume servers in: & $\mathbf{2 0 0 7}$ & $\mathbf{2 0 0 8}$ & $\mathbf{2 0 0 9}$ & $\mathbf{2 0 1 0}$ & $\mathbf{2 0 1 1}$ \\
\hline Server closets & 1,456 & 1,439 & 1,386 & 1,296 & 1,326 \\
\hline Server rooms & 1,465 & 1,472 & 1,427 & 1,334 & 1,371 \\
\hline Localized data centers & 1,465 & 1,471 & 1,426 & 1,334 & 1,371 \\
\hline Mid-tier data centers & 1,465 & 1,471 & 1,426 & 1,334 & 1,371 \\
\hline Enterprise-class data centers & 1,465 & 1,471 & 1,426 & 1,334 & 1,371 \\
\hline \multicolumn{7}{|c|}{ State-of-the-art scenario } \\
\hline Volume servers in: & $\mathbf{2 0 0 7}$ & $\mathbf{2 0 0 8}$ & $\mathbf{2 0 0 9}$ & $\mathbf{2 0 1 0}$ & $\mathbf{2 0 1 1}$ \\
\hline Server closets & 1,485 & 1,471 & 1,424 & 1,315 & 1,349 \\
\hline Server rooms & 1,495 & 1,573 & 1,586 & 1,424 & 1,485 \\
\hline Localized data centers & 1,495 & 1,572 & 1,585 & 1,424 & 1,485 \\
\hline Mid-tier data centers & 1,495 & 1,572 & 1,585 & 1,424 & 1,485 \\
\hline Enterprise-class data centers & 1,495 & 1,572 & 1,585 & 1,424 & 1,485 \\
\hline
\end{tabular}

Table A4-13 summarizes the projections for the average UEC of mid-range and high-end servers in all three scenarios. The average UEC values for mid-range servers are based on the assumption of $100 \%$ power management utilization in all three scenarios as indicated in Table 35.

Table A4-13. Projected Average UEC (in kWh/year) for Mid-range and High-end Servers, Alternative Scenarios, 2007 to 2011

\begin{tabular}{|l|c|c|c|c|c|}
\hline \multicolumn{7}{|c|}{ All alternative scenarios } \\
\hline Server class & $\mathbf{2 0 0 7}$ & $\mathbf{2 0 0 8}$ & $\mathbf{2 0 0 9}$ & $\mathbf{2 0 1 0}$ & $\mathbf{2 0 1 1}$ \\
\hline Mid-range & 4,921 & 5,467 & 6,152 & 6,649 & 7,185 \\
\hline High-end & 76,295 & 81,624 & 86,849 & 92,662 & 98,864 \\
\hline
\end{tabular}


Table A4-14 summarizes the total projected energy use of U.S. servers by space type for the all three alternative efficiency scenarios, based on the installed server base and average UEC assumptions described above. When compared to Table A4-7, the projections in Table A4-14 suggest that the total energy use of U.S. servers can be reduced by a significant fraction in all three efficiency scenarios.

Table A4-14. Projected Total Energy Use of U.S. Servers (in billion kWh/year) by Space Type, Alternative Scenarios, 2007 to 2011

\begin{tabular}{|c|c|c|c|c|c|}
\hline \multicolumn{6}{|c|}{ Improved operation scenario } \\
\hline Space type & 2007 & 2008 & 2009 & 2010 & 2011 \\
\hline Server closet & 2.8 & 3.1 & 3.3 & 3.5 & 3.7 \\
\hline Server room & 3.7 & 4.3 & 4.9 & 5.5 & 6.3 \\
\hline Localized data center & 3.6 & 4.1 & 4.7 & 5.2 & 5.9 \\
\hline Mid-tier data center & 3.3 & 3.7 & 4.2 & 4.7 & 5.3 \\
\hline Enterprise-class data center & 7.6 & 8.6 & 9.6 & 10.6 & 11.8 \\
\hline Total & 21.0 & 23.8 & 26.7 & 29.4 & 32.9 \\
\hline \multicolumn{6}{|c|}{ Best practice scenario } \\
\hline Space type & 2007 & 2008 & 2009 & 2010 & 2011 \\
\hline Server closet & 2.6 & 2.5 & 2.4 & 2.3 & 2.3 \\
\hline Server room & 3.0 & 3.0 & 2.9 & 2.7 & 2.8 \\
\hline Localized data center & 3.0 & 3.0 & 2.9 & 2.8 & 2.9 \\
\hline Mid-tier data center & 2.7 & 2.7 & 2.6 & 2.5 & 2.6 \\
\hline Enterprise-class data center & 6.6 & 6.5 & 6.5 & 6.3 & 6.5 \\
\hline Total & 17.9 & 17.7 & 17.3 & 16.6 & 17.1 \\
\hline \multicolumn{6}{|c|}{ State-of-the-art scenario } \\
\hline Space type & 2007 & 2008 & 2009 & 2010 & 2011 \\
\hline Server closet & 2.5 & 2.3 & 2.1 & 1.9 & 1.8 \\
\hline Server room & 2.8 & 2.4 & 1.9 & 1.3 & 1.3 \\
\hline Localized data center & 2.8 & 2.5 & 2.1 & 1.6 & 1.6 \\
\hline Mid-tier data center & 2.5 & 2.2 & 1.9 & 1.5 & 1.4 \\
\hline Enterprise-class data center & 6.1 & 5.6 & 5.0 & 4.3 & 4.2 \\
\hline Total & 16.6 & 14.9 & 13.0 & 10.6 & 10.3 \\
\hline
\end{tabular}

Estimation of Energy Use for Storage Devices and Network Equipment

Due to lack of data on the likely energy efficiency trends associated with network equipment over the next five years, it was assumed that the network equipment energy use trends established in the historical trends scenario would be valid for all scenarios considered in this study. The projections for the energy use of network equipment by space type for the historical trends scenario were previously summarized in Table A4-9.

The energy use projections associated with enterprise storage devices for the improved operation scenario were assumed to be the same as the energy use projections for the current efficiency 
trends scenario in Table A4-8. This assumption was made because no changes to enterprise storage systems were assumed between these two scenarios.

As indicated in Table 3-5, for the best practice and state-of-the-art scenarios it was assumed that reductions in physical storage devices via virtualization would be pursued as an energy savings strategy. As for servers, virtualization of storage devices allows for the replacement of many storage devices operating at low utilization rates with fewer storage devices operating at higher utilization rates. Energy savings are realized because fewer drives need to be kept "spinning" to meet the data storage and access needs of the enterprise.

According to industry data, the current utilization rates for enterprise storage devices range from $25 \%$ to $40 \%$; however, with storage virtualization, average utilization rates of $60 \%$ are possible (Battles et al. 2007). In the best practice scenario analysis, a moderate 1.5 to 1 physical storage reduction ratio in 2011 was assumed, based on average storage utilization rates increasing from $40 \%$ to $60 \%$ as indicated by Battles et al. (2007). It was assumed that $80 \%$ of the installed base of external storage would be available for virtualization (meaning that $20 \%$ of the installed base has already virtualized). In the state-of-the-art scenario analysis, a more aggressive $\sim 2.5$ to 1 physical storage reduction ratio in 2011 was assumed, based on average storage utilization rates increasing from $25 \%$ to $60 \%$ as indicated by Battles et al. (2007). Again, it was assumed that this ratio would be applicable to $80 \%$ of the installed base. In each scenario, a linear increase in the physical storage reduction ratio each year up to the assumed 2011 value was assumed.

The resulting projections for the total annual energy consumed by enterprise storage devices for the best practice and state-of-the-art scenarios are summarized in Table A4-15. Comparing the projections in Table A4-15 to the current efficiency trends scenario projections in Table A4-8 reveals that significant energy savings may be achievable through physical storage reduction.

Table A4-15. Projected Total Energy Use of U.S. Enterprise Storage Devices (in billion kWh/year) by Space Type, Best Practice and State-of-the-Art Scenarios, 2007 to 2011

\begin{tabular}{|c|c|c|c|c|c|}
\hline \multicolumn{6}{|c|}{ Best practice } \\
\hline Space type & 2007 & 2008 & 2009 & 2010 & 2011 \\
\hline Server closet & 0.0 & 0.0 & 0.0 & 0.0 & 0.0 \\
\hline Server room & 0.0 & 0.0 & 0.0 & 0.0 & 0.0 \\
\hline Localized data center & 1.0 & 1.2 & 1.4 & 1.8 & 2.0 \\
\hline Mid-tier data center & 0.9 & 1.1 & 1.3 & 1.6 & 1.8 \\
\hline Enterprise-class data center & 1.8 & 2.2 & 2.6 & 3.2 & 3.7 \\
\hline Total & 3.60 & 4.45 & 5.36 & 6.56 & 7.51 \\
\hline \multicolumn{6}{|c|}{ State-of-the-art } \\
\hline Space type & 2007 & 2008 & 2009 & 2010 & 2011 \\
\hline Server closet & 0.0 & 0.0 & 0.0 & 0.0 & 0.0 \\
\hline Server room & 0.0 & 0.0 & 0.0 & 0.0 & 0.0 \\
\hline Localized data center & 0.8 & 1.0 & 1.1 & 1.3 & 1.4 \\
\hline Mid-tier data center & 0.8 & 0.9 & 1.0 & 1.1 & 1.3 \\
\hline Enterprise-class data center & 1.6 & 1.8 & 2.0 & 2.4 & 2.7 \\
\hline Total & 3.16 & 3.60 & 4.08 & 4.79 & 5.46 \\
\hline
\end{tabular}




\section{Estimation of Energy Used by Site Infrastructure Systems}

The assessments of site infrastructure system efficiency in this report focuses primarily on estimating how the PUE ratio would be likely to change over the next five years given representative energy efficiency improvements for each alternative efficiency scenario. The assumed maximum achievable PUE ratios by space type for each alternative efficiency scenario are summarized in Table A4-16. The assumptions behind each of these projections are discussed below.

Table A4-16. Assumptions for Maximum Achievable PUE Ratios in 2011 by Space Type

\begin{tabular}{|l|c|c|c|}
\hline \multirow{2}{*}{ Space type } & \multicolumn{3}{|c|}{2011 maximum achievable PUE ratio } \\
\cline { 2 - 4 } & $\begin{array}{c}\text { Improved } \\
\text { operation }\end{array}$ & $\begin{array}{c}\text { Best } \\
\text { practice }\end{array}$ & $\begin{array}{c}\text { State-of- } \\
\text { the- art }\end{array}$ \\
\hline Server closet & 1.7 & 1.7 & 1.7 \\
\hline Server room & 1.7 & 1.7 & 1.7 \\
\hline Localized data center & 1.7 & 1.3 & 1.3 \\
\hline Mid-tier data center & 1.7 & 1.3 & 1.3 \\
\hline Enterprise-class data center & 1.7 & 1.3 & 1.2 \\
\hline
\end{tabular}

Improved operation scenario

This scenario assumes essentially the same site infrastructure systems as would be in place as in the current efficiency trends scenario. This equipment would typically include:

- $95 \%$ efficient transformers

- $80 \%$ efficient UPS

- Air cooled direct exchange system chiller

- Constant speed fans

- Humidification control

- Redundant air handling units

It was assumed that representative measures for improved operation of site infrastructure systems would involve strategically orienting equipment and managing airflow to reduce air resistance and eliminate short circuiting (i.e., the mixing of hot and cold air within the room). Reducing air resistance lowers the system fan power, while eliminating short-circuiting allows the supply temperature to be raised, which in turn lowers the chiller power draw. These measures have the potential to reduce fan energy use by $20-25 \%$ and can also reduce chiller energy use by $20 \%$ (Eubank et al. 2003).

A shift from the current trends scenario PUE ratio of 1.9 to a PUE ratio of 1.7 for the improved operational management scenario matches well with these anticipated fan and chiller savings. Also, a PUE ratio of 1.7 matches the theoretical ratio derived from typical equipment energy use presented in Table A4-17. This theoretical ratio is expected since the improved operational 
management scenario assumes that the majority of inefficiencies not associated with the equipment have been removed.

Table A4-17. Equipment Contributions, Improved Operation Scenario

\begin{tabular}{|l|c|}
\hline 2011 ratio of total energy use to IT equipment energy use & $\mathbf{1 . 7}$ \\
\hline Approximate equipment contribution to ratio & \\
\hline IT equipment & 1.0 \\
\hline Transformer losses & 0.05 \\
\hline UPS losses & 0.2 \\
\hline Chiller & 0.3 \\
\hline Fans & 0.13 \\
\hline Lighting & 0.02 \\
\hline
\end{tabular}

Table A4-18 summarizes the assumed PUE ratio by space type and year for the improved operation scenario projections. It was assumed that the PUE ratio would improve in a linear fashion from the 2006 PUE ratio of 2.0 to the maximum achievable PUE ratio in Table A4-16 for all space types over the five-year analysis period to allow for gradual adoption rates of improved operation efficiency practices.

Table A4-18. Assumed PUE Ratio by Space Type and Year, Improved Operation Scenario, 2007 to 2011

\begin{tabular}{|r|c|c|c|c|c|}
\hline Space type & $\mathbf{2 0 0 7}$ & $\mathbf{2 0 0 8}$ & $\mathbf{2 0 0 9}$ & $\mathbf{2 0 1 0}$ & $\mathbf{2 0 1 1}$ \\
\hline Server closet & 1.94 & 1.88 & 1.82 & 1.76 & 1.70 \\
\hline Server room & 1.94 & 1.88 & 1.82 & 1.76 & 1.70 \\
\hline Localized data center & 1.94 & 1.88 & 1.82 & 1.76 & 1.70 \\
\hline Mid-tier data center & 1.94 & 1.88 & 1.82 & 1.76 & 1.70 \\
\hline Enterprise-class data center & 1.94 & 1.88 & 1.82 & 1.76 & 1.70 \\
\hline
\end{tabular}

\section{$\underline{\text { Best practice scenario }}$}

It was assumed that a best practice facility would have performance equal to the most energy efficient facilities identified in recent benchmarking studies of 22 data centers performed by Lawrence Berkeley National Laboratory (Tschudi et al. 2004; Greenberg et al. 2006). The best PUE ratios identified in these benchmarking studies were around 1.3. Infrastructure systems in such facilities use proven energy efficient technologies that commonly include:

- $98 \%$ efficient transformers

- $\quad 90 \%$ efficient UPS

- Variable-speed drive chiller with economizer cooling or water side free cooling

- Variable-speed fans and pumps

- Redundant air handling units

Table A4-19 summarizes the contributions of typical equipment energy use and estimates a theoretical PUE ratio of 1.21 (Rumsey 2007), which is in reasonable agreement with the PUE 
ratio of 1.3 observed at "best in class" data centers. The representative equipment assumed for the best practices scenario would not be appropriate for small data centers, and therefore is not applied to server closets and server rooms in Table A4-16.

Table A4-19. Equipment Contributions, Best Practice Scenario

\begin{tabular}{|l|c|}
\hline 2011 ratio of total energy use to IT equipment energy use & $\mathbf{1 . 3}$ \\
\hline Approximate equipment contribution to ratio & \\
\hline IT equipment & 1.0 \\
\hline Transformer losses & 0.03 \\
\hline UPS losses & 0.1 \\
\hline Chiller & 0.1 \\
\hline Fans and Lighting & 0.05 \\
\hline
\end{tabular}

Table A4-20 summarizes the assumed PUE ratio by space type and year for the best practice scenario projections. It was assumed that the PUE ratio would improve in a linear fashion from the 2006 PUE ratio of 2.0 to the maximum achievable PUE ratio in Table A4-16 for 50\% of the U.S. facilities in each space type. The assumption of applicability to only $50 \%$ of U.S. facilities acknowledges that the aggressive improvements associated with the best practice scenario may only be feasible during major equipment upgrades, facility expansions, or new facility construction.

Table A4-20. Assumed PUE Ratio by Space Type and Year, Best Practice Scenario, 2007 to 2011

\begin{tabular}{|r|c|c|c|c|c|}
\hline Space type & $\mathbf{2 0 0 7}$ & $\mathbf{2 0 0 8}$ & $\mathbf{2 0 0 9}$ & $\mathbf{2 0 1 0}$ & $\mathbf{2 0 1 1}$ \\
\hline Server closet & 1.94 & 1.88 & 1.82 & 1.76 & 1.70 \\
\hline Server room & 1.94 & 1.88 & 1.82 & 1.76 & 1.70 \\
\hline Localized data center & 1.90 & 1.80 & 1.70 & 1.60 & 1.50 \\
\hline Mid-tier data center & 1.90 & 1.80 & 1.70 & 1.60 & 1.50 \\
\hline Enterprise-class data center & 1.90 & 1.80 & 1.70 & 1.60 & 1.50 \\
\hline
\end{tabular}

\section{$\underline{\text { State-of-the-art scenario }}$}

It was assumed that representative infrastructure equipment for a state of the facility would include emerging energy efficient technologies such as liquid cooling (instead of air), DC power distribution to reduce UPS losses, and distributed generation using combined heat and power (CHP). A cooling tower with variable speed pumps to rack coils would reduce cooling system power to roughly $0.15 \mathrm{~kW} /$ ton (Rumsey 2007). Typical equipment in a state-of-the-art facility would include:

- $98 \%$ efficient transformers

- $95 \%$ efficient UPS

- liquid cooling to the racks

- cooling tower

- variable-speed drive pumps 


\section{- $\mathrm{CHP}$}

Table A4-21 summarizes the contributions of typical equipment energy use and estimates a theoretical PUE ratio of 1.14 (Rumsey 2007), which was rounded up to 1.2 to account for location variability in economizer use and any other potential inefficiencies. The representative equipment assumed for the state-of-the-art scenario would only be appropriate for very large data centers, and therefore was only applied to enterprise-class data centers in Table A4-16.

Table A4-21. Equipment Contributions, State-of-the-Art Scenario

\begin{tabular}{|l|l|}
\hline 2011 ratio of total energy use to IT equipment energy use & $\mathbf{1 . 2}$ \\
\hline Approximate equipment contribution to ratio & \\
\hline IT equipment & 1.0 \\
\hline Transformer losses & 0.03 \\
\hline UPS losses & 0.05 \\
\hline Pumps and fans & 0.04 \\
\hline Lighting & 0.02 \\
\hline
\end{tabular}

Table A4-22 summarizes the assumed PUE ratio by space type and year for the state-of-the-art scenario projections. It was assumed that the PUE ratio would improve in a linear fashion from the 2006 PUE ratio of 2.0 to the maximum achievable PUE ratio in Table A4-16 for 50\% of the U.S. facilities in each space type. The assumption of applicability to only $50 \%$ of U.S. facilities acknowledges that the aggressive improvements associated with the state-of-the-art scenario may

only be feasible during major equipment upgrades, facility expansions, or new facility construction.

Table A4-22. Assumed PUE Ratio by Space Type and Year, State-of-the-Art Scenario, 2007 to 2011

\begin{tabular}{|r|c|c|c|c|c|}
\hline Space type & $\mathbf{2 0 0 7}$ & $\mathbf{2 0 0 8}$ & $\mathbf{2 0 0 9}$ & $\mathbf{2 0 1 0}$ & $\mathbf{2 0 1 1}$ \\
\hline Server closet & 1.94 & 1.88 & 1.82 & 1.76 & 1.70 \\
\hline Server room & 1.94 & 1.88 & 1.82 & 1.76 & 1.70 \\
\hline Localized data center & 1.90 & 1.80 & 1.70 & 1.60 & 1.50 \\
\hline Mid-tier data center & 1.90 & 1.80 & 1.70 & 1.60 & 1.50 \\
\hline Enterprise-class data center & 1.89 & 1.78 & 1.67 & 1.56 & 1.45 \\
\hline
\end{tabular}

The resulting projections for the total energy use attributable to infrastructure systems by space type for the three alternative scenarios (based on the ratios presented in Tables A4-18, A4-20, and A4-22) are summarized in Table A4-23. 
Table A4-23. Projected Total Energy Use of Site Infrastructure Systems (in billion kWh/year) by Space Type, Alternative Scenarios, 2007 to 2011

\begin{tabular}{|c|c|c|c|c|c|}
\hline \multicolumn{6}{|c|}{ Improved operation scenario } \\
\hline Space type & 2007 & 2008 & 2009 & 2010 & 2011 \\
\hline Server closet & 2.8 & 2.9 & 2.9 & 2.9 & 2.8 \\
\hline Server room & 4.1 & 4.5 & 4.8 & 5.0 & 5.3 \\
\hline Localized data center & 5.0 & 5.5 & 6.0 & 6.5 & 6.9 \\
\hline Mid-tier data center & 4.5 & 5.0 & 5.4 & 5.9 & 6.2 \\
\hline Enterprise-class data center & 10.2 & 11.1 & 11.9 & 12.8 & 13.4 \\
\hline Total & 26.6 & 29.0 & 31.1 & 33.0 & 34.6 \\
\hline \multicolumn{6}{|c|}{ Best practice scenario } \\
\hline Space type & 2007 & 2008 & 2009 & 2010 & 2011 \\
\hline Server closet & 2.6 & 2.4 & 2.2 & 1.9 & 1.8 \\
\hline Server room & 3.5 & 3.3 & 3.1 & 2.9 & 2.9 \\
\hline Localized data center & 4.2 & 3.9 & 3.7 & 3.4 & 3.1 \\
\hline Mid-tier data center & 3.7 & 3.6 & 3.3 & 3.0 & 2.8 \\
\hline Enterprise-class data center & 8.7 & 8.2 & 7.6 & 7.0 & 6.3 \\
\hline Total & 22.7 & 21.4 & 19.9 & 18.2 & 16.8 \\
\hline \multicolumn{6}{|c|}{ State-of-the-art scenario } \\
\hline Space type & 2007 & 2008 & 2009 & 2010 & 2011 \\
\hline Server closet & 2.5 & 2.2 & 1.9 & 1.6 & 1.5 \\
\hline Server room & 3.2 & 2.8 & 2.3 & 1.9 & 1.8 \\
\hline Localized data center & 3.8 & 3.3 & 2.8 & 2.4 & 2.1 \\
\hline Mid-tier data center & 3.5 & 3.0 & 2.6 & 2.1 & 1.9 \\
\hline Enterprise-class data center & 8.0 & 6.9 & 5.9 & 4.9 & 4.2 \\
\hline Total & 21.0 & 18.3 & 15.5 & 12.9 & 11.5 \\
\hline
\end{tabular}

\section{References}

Advanced Micro Devices (AMD). 2006. Power and Cooling in the Data Center. AMD Enterprise White Paper 34246C. http://enterprise.amd.com/Downloads/34146A_PC_WP_en.pdf

Bailey, M., M. Eastwood, T Grieser, L. Borovick, V. Turner, and R.C. Gray. 2007. Special Study: Data Center of the Future. IDC. IDC \#06C4799. April.

Battles, Brett, Cathy Belleville, Susan Grabau, Judith Maurier. 2007. Reducing Data Center Power Consumption Through Efficient Storage. Network Appliance, Sunnyvale, California. February. http://www.netapp.com/ftp/wp-reducing-datacenter-power-consumption.pdf

Dietrich, J. 2007. Personal communication with Jay Dietrich of IBM. March 19, 2007.

Greenberg, S., E. Mills, W. Tshudi, P. Rumsey, and B. Myatt. 2006. "Best Practices for Data Centers: Results from Benchmarking 22 Data Centers.” Proceedings of the 2003 ACEEE Summer Study on Energy Efficiency in Buildings. Asilomar, California. August. 
IDC. 2007a. IDC's Worldwide Installed Base Forecast, 2007-2010. Spreadsheet Provided to Lawrence Berkeley National Laboratory. March.

IDC. 2007b. "Virtualization and Multicore Innovations Disrupting the Worldwide Server Market, According to IDC .” Framingham, MA: IDC. Press Release. March 20. http://www.idc.com/getdoc.jsp?containerId=prUS20609907

Koomey, J.G. 2007. Estimating Total Power Consumption by Servers in the U.S. and the World. Report prepared for Advanced Micro Devices. February 15.

http://enterprise.amd.com/Downloads/svrpwrusecompletefinal.pdf

Nordman, B. 2005. Metrics of IT Equipment - Computing and Energy Performance. Lawrence Berkeley National Laboratory, Berkeley, California. http://eetd.lbl.gov/Controls/metricsMARCH10.pdf

Osterberg, K. 2007. Personal communication with Ken Osterberg of Seagate Technology. March 13, 2007.

Rumsey, P. 2007. Personal communication with Peter Rumsey of Rumsey Engineering. March 7, 2007.

Tschudi, W., T. Xu, D. Sartor, and J. Stein. 2004. High-Performance Data Centers: A Research Roadmap. Lawrence Berkeley National Laboratory, Berkeley, California. LBNL-53483.

U.S. Environmental Protection Agency (EPA). 2007. Working Group Notes from the EPA Technical Workshop on Energy Efficient Servers and Datacenters. Santa Clara Convention Center. February 16. 


\section{Appendix 5. Summary of current state energy efficiency programs}

\section{National Policies}

Energy Efficient Commercial Buildings Tax Deduction: Created by EPACT05, buildings placed in service from 1/1/06 through 12/31/08 are eligible for a tax deduction of \$1.80/square ft to owners of new or existing buildings who install improvements to interior lighting, building envelope or the HVAC system that reduce a building's total energy and power cost by $50 \%$ or more compared to an ASHRAE Standard 90.1-2001 reference building. Deductions of \$0.60/sq $\mathrm{ft}$ are available for those owners that only retrofit one of the three categories but achieve 16 2/3\% energy reductions compared to ASHRAE 90.1-2001.

Since data centers are excluded from ASHRAE HVAC requirements, they won't be able to receive that part of the deduction.

http://www.energytaxincentives.org

Federal Agency Energy Reduction Goals: EO 13423, signed in January 2007, mandates that all federal agencies reduce their energy intensity by 3\% a year, or 30\% by 2015, relative to its 2005 baseline energy consumption.

Energy Saving Performance Contract (ESPC) authorization: Federal agencies were reauthorized to engage in ESPCs at the start of Fiscal Year 2005 (authority for the program had lapsed in 2003). In FY 2005, 15 of the 20 ESPCs were awarded by the Department of Defense, accounting for about 70 per cent of the financial savings reaped by the government through ESPCs and about two-thirds of the energy savings. ${ }^{2}$ Although ESPCs do not typically include retrofits to data centers, if agencies began approaching energy service companies (ESCOs) about data center improvements, the ESCOs would certainly begin offering that service. This would require overcoming agencies' (especially those agencies with sensitive operations like Homeland Security or the Department of Defense) reticence to share information or data on their data centers due to security concerns.

SAVEnergy Audits: Through the Federal Energy Management Program (FEMP), agencies can request an energy audit by a FEMP-qualified engineer. The auditor will conduct a “comprehensive examination” of a federal facility or building's energy systems. Traditionally these audits have not included analyses of data centers, but there is no reason they could not be expanded to do so. Funding for these audits were eliminated in FY 2006, however, although the contracts still exist. Other agencies can still fund audits using the Department of Energy contract.

$2 \quad$ Federal Energy Management Program, Annual Report to Congress on Federal Government Energy Management and Conservation Programs Fiscal Year 2005, September 26, 2006, http://www1.eere.energy.gov/femp/pdfs/annrep05.pdf. 
http://www1.eere.energy.gov/femp/services/assessments savenergy.html

\section{Energy-Efficient Project Funding}

\section{Arizona}

The Arizona Municipal Energy Management Program awards grants to encourage and assist in the development and implementation of energy management programs by helping with planning and providing the necessary basic tools, staff training and technical assistance. Arizona cities, towns, counties, improvement districts, and Indian tribes with populations under 70,000 are eligible. The Energy Office in the Arizona Department of Commerce funds these grants.

http://www.commerce.state.az.us/Energy/default.asp

The Arizona Energy Conservation Savings Reinvestment Plan for the City of Phoenix, started in 1984, provides secure and long-term loans for energy-efficiency initiatives under the Energy Management Program. Under this plan, 50 percent of all documented energy savings (up to $\$ 750,000$ ) must be reinvested in further efficiency improvements. All municipal departments in Phoenix are eligible. Eligible projects include upgrading lighting, motors and chillers, among other upgrades.

http://www.iclei.org/index.php?id=1677\&0=

\section{California}

California’s Energy Efficiency Financing Program offers loans to public schools, public hospitals, cities, counties, special districts, and public care institutions (public only). Eligible projects are those with proven energy savings, such as lighting and HVAC efficiency improvements. The Program has a \$40 million endowment, with a maximum loan of \$3 million per application. There is no minimum loan amount. The projects must be technically or economically feasible and must have a simple payback of 9.8 years or less, based on energy savings. Additionally, the Energy Commission provides technical assistance to help customers identify ways to save energy costs and to encourage the most efficient use of energy in their facilities. The majority of these programs are for public agencies. The Bright Schools Program helps public K-12 school districts and non-profit schools reduce energy costs in their facilities. The Energy Partnership Program targets the same entities as the loan program does and also nonprofit schools, hospitals, colleges and public care facilities. Both the Bright Schools and Energy Partnership Programs pays a portion of the consultant's cost associated with preparing a report — often this cost is sufficient to analyze one or more facilities.

http://www.energy.ca.gov/efficiency/public_programs.html 


\section{Connecticut}

Connecticut's “Act Concerning Energy Independence,” passed in 2005, offers incentives for businesses to produce and conserve energy through monetary grants, low-interest financing, and reduced back-up electric rates, among other measures.

http://www.cl-p.com/clpcommon/pdfs/clmbus/target/blueprint_cutsheet.pdf

The Connecticut Energy Efficiency Fund (CEEF) offers financial incentives to business customers making energy-efficiency improvements, especially for peak demand reduction. These incentives are designed to cover the incremental cost of energy-efficient equipment, and can add up to as much as $\$ 300,000$ annually.

http://www.ctsavesenergy.org/

\section{Idaho}

The Idaho Energy Conservation Loan Program, started in 1987, provides loans with 4\% annual interest for energy conservation measures and the promotion of renewable resources. The Program was originally funded by the settlements Idaho received from Exxon and Stripper Well. Its endowment is $\$ 5,015,000$, and individual loans are capped at $\$ 10,000$ for residential projects and $\$ 100,000$ for commercial, governmental, agricultural and school, hospital or health care facility projects, and all projects must have a payback period of no more than five years from energy savings.

http://www.idwr.state.id.us/

\section{Iowa}

Iowa's Energy Bank provides technical and financial assistance to public and nonprofit facilities for cost-effective energy efficiency improvements. Like an ESPC, it uses the energy cost savings to finance the improvements. It's focused on public and private schools, private colleges, hospitals and local governments. A six-month, interest-free loan is available for the initial energy audit and engineering analysis.

http://www.iowadnr.com/energy/ebank/index.html 


\section{Kansas}

A bill signed in April 2006 allows a municipality or state agency to enter into a contract or leasepurchase agreement for qualified energy conservation measures.

http://www.kslegislature.org/bills/2006/2602.pdf

\section{Maryland}

The Maryland Community Energy Loan Program, founded in 1989, offers loans to nonprofits and local governments, including private and public schools for expenses associated with the identification and implementation of energy-efficiency improvements. Eligible projects will have a payback of no more than seven years. The Fund's endowment is \$3.2 million, and originally came from the Oil Overcharge Fund. The loans range from \$30,000 to \$400,000.

http://www.energy.state.md.us/programs/government/communityenergyloan.htm

The Maryland State Agency Loan Program, found in 1991, provides no-interest loans to state agencies for energy-efficiency improvements. The maximum payback period for eligible projects is ten years, and the maximum loan is $\$ 600,000$.

http://www.energy.state.md.us/programs/government/stateagencyloan.htm

\section{Mississippi}

The Mississippi Energy Investment Loan Program, founded in 1989, provides loans at 3\% below the prevailing Prime Interest rate to individuals, partnerships and corporations for retrofit projects or for the design and development of innovative energy conservation processes. The Program's endowment is $\$ 6$ million and the size of its loans range from $\$ 15,000$ to $\$ 300,000$. The maximum payback period is ten years for eligible projects.

http://www.mississippi.org/content.aspx?url=/page/2913\&

\section{Missouri}

Missouri's Energy Loan Program, founded in 1990, offers below-market interest rate loans to schools and local governments for energy conservation projects. The loans range from $\$ 5,000$ to \$2 million.

http://www.dnr.mo.gov/eiera/energy-efficiency-loans.htm 


\section{Montana}

The Montana State Buildings Energy Conservation Program, founded in 1989, offers loans to state agencies for the identification and implementation of cost-effective energy-efficiency improvements. The program is funded through the sale of general obligation bonds. Eligible programs must have a ten year payback period. There is no maximum loan size.

http://www.deq.state.mt.us/Energy/buildings/StateBuildings.asp

\section{Nebraska}

Nebraska's Dollar and Energy Saving Loan Program offers low-interest financing for many typical home, building or system energy improvements. Financing is also available for other types of efficiency improvements, such as alternate fuel vehicle or fueling facility, telecommunications equipment or waste minimization. The program's endowment is $\$ 23$ million, and the loan size ranges from $\$ 35,000$ to $\$ 175,000$ depending on the type of project.

http://www.neo.ne.gov/loan/

\section{New Hampshire}

The New Hampshire Building Energy Conservation Initiative was created in 1999 and will run through 2019. It offers 3.85\% interest rate loans to state agencies for the construction and implementation of energy-efficient building improvements. The endowment is \$25 million and the maximum payback period is ten years.

http://nh.gov/oep/programs/energy/beci.htm

\section{New York}

The New York Energy Smart Loan Fund will offer loans with an interest rate reduction of up to 4\% off normal interest rates through July 31, 2007 (extended from the initial expiration date of June 30, 2006) for facilities installing energy-efficiency improvements and/or renewable technologies. For certain customers in the Con Edison service territory, the interest rate reduction is up to $6.5 \%$. Residential, multifamily (i.e. apartment buildings) and commercial loans are all available. The size of the loan varies depending on the type of loan.

http://www.nyserda.org/loanfund/default.asp

The NYSERDA New Construction Program provides technical assistance and financial incentives to design teams and building owners. NCP offers direct technical assistance design incentives and capital cost incentives based on improved building energy efficiency performance. Incentives are also available for building commissioning services, green buildings, 
peak-load reduction, energy benchmarking, and advanced solar and daylighting systems. Current funding opportunities are available under program opportunity notice (PON) number 1155 with an expiration date of March 31, 2008. http://www.nyserda.org/programs/New_Construction The New York Energy Smart Flexible Technical Assistance (“FlexTech”) program provides cost-share funding opportunities to provide a variety of technical assistance services to New York State companies, custom-tailored to meet cost-effective energy-related needs. NYSERDA has contracted with engineering firms that were competitively selected through an RFP process to provide services such as engineering feasibility studies, process improvement, rate analysis and load shapes, and retro-commissioning. http://www.nyserda.org/programs/flextech.asp

\section{Oklahoma}

The Oklahoma Community Energy Education Municipal Program, founded in 1995, offers lowinterest loans to counties, cities and towns in Oklahoma for energy-efficiency improvements. The endowment is $\$ 1$ million and loans generally do not exceed $\$ 150,000$.

http://staging.okcommerce.gov/test1/dmdocuments/Community_Energy_Education_Management_Loan_Program_Guidance_1312062064.pdf

The Oklahoma Energy Loan Fund for Schools, founded in 1998, offers low-interest loans to K12 schools for energy-efficiency improvements. The endowment is \$1 million, and the maximum loan is $\$ 100,000$. The payback period for eligible projects ranges from 18 months to seven years.

http://staging.okcommerce.gov/test1/dmdocuments/Energy_Loan_Fund_for_Schools_Program_Guidance_Application_1312062065.pdf

\section{Oregon}

The Oregon Energy Loan Program, created in 1979, offers low-interest, fixed rate loans to individuals, schools, cities, counties, special districts, state and federal agencies, public corporations, cooperatives, tribes and non-profits for energy conservation, renewable energy, alternative fuels or recycled product production. The Program is funded through Oregon general obligation bonds, and offers loans ranging from \$20,000 to \$11 million. The payback period for eligible projects ranges from five to 15 years.

http://egov.oregon.gov/ENERGY/LOANS/selphm.shtml 


\section{Pennsylvania}

The Pennsylvania Sustainable Energy Funds, established in 2000, offer loans for programs that promote energy-efficiency and conservation or renewable/clean energy. There are four funds, established after deregulation, and each one run by one of the State's four major utilities (GPU Energy, PECO Energy, PP\&L and Allegheny Power/West Penn Power Company. Combined, the four funds have an endowment of approximately $\$ 83.5$ million.

http://www.puc.state.pa.us/electric/greenclean/Green_Clean.htm\#Sustainable\%20Energy\%20Funds

\section{South Carolina}

The South Carolina Conserfund Loan Program offers 5\% maximum interest rate loans to state and local governments, schools and colleges, hospitals and other nonprofit organizations for energy-efficiency improvements. The Program's endowment comes from the Stripper Well Settlement funds. The loans range from $\$ 25,000$ to $\$ 500,000$. The payback period for eligible programs can be as large as ten years.

http://www.energy.sc.gov/index.aspx?m=7\&t=48\&h=180

\section{Tennessee}

The Tennessee Local Government Loan Program, started in 1991, offers 3\% interest rate loans to local government agencies including public school systems for energy-efficiency improvements. The endowment is provided by the Petroleum Violation Escrow fund. Loans up to \$500,000 are offered. The maximum payback period for eligible programs is seven years.

The Tennessee Small Business Energy Loan Program, founded in 1988, offers 3\% interest rate loans to small businesses (less than 300 employees or less than 3.5 million dollars in annual gross sales or receipts) for energy-efficiency upgrades in their buildings, plants and manufacturing processes. The endowment is provided by the Petroleum Violation Escrow fund. Loans up to $\$ 100,000$ are offered. The maximum payback period for eligible programs is seven years.

http://www.state.tn.us/ecd/energy_loans.htm

\section{Texas}

The Texas LoanSTAR Revolving Loan Program, founded in 1989, offers loans to state agencies, institutions of higher learning, school districts and local governments for energy-efficiency 
retrofits. The fund endowment is $\$ 98$ million and comes from the 1976 oil overcharge funds. Loans from $\$ 10,000$ to $\$ 5$ million are offered. The maximum payback period is ten years.

http://www.seco.cpa.state.tx.us/ls.htm

\section{Energy-Saving Performance Contracts}

ESPCs are allowed for all public agencies and actors in: AL, AK, AZ, CA, CO, CT, FL, HI, ID, IN, IA, KS, KY, LA, ME, MA, MS, MO, NE, NV, NM, NY, ND, OR, PA, RI, SC, SD, TN, TX, UT, VA, WA, and WV.

They are allowed for certain publicly owned buildings in: AR, DE, GA, IL, MD, MI, MN, NH, NJ, NC, OH, OK, and WI.

No authorization is allowed for any publicly owned buildings in VT and WY.

http://www.ornl.gov/info/esco/legislation/

\section{Washington}

In 2001, legislation was passed in Washington that required state facilities to conduct energy audits to identify potential energy-saving opportunities, and to explore ESPCs as their first option to capture those savings. Although all of the initial audits should be completed by now, the state has since created an energy performance contracting program specifically for state agencies, colleges and universities, cities and towns, counties, school districts, ports, libraries, hospitals, and health districts which provides free energy audits.

http://www.ga.wa.gov/EAS/epc/espc.htm

\section{Energy-Efficiency Portfolio Standards}

In general, energy-efficiency portfolio standards (EEPS) do not figure to have a direct impact on data center operations. However, in as much as data center load is becoming a problem for utilities looking to lower their peak load, especially in the pacific northwest, data centers could offer large and relatively easy energy-savings opportunities for electric utilities who have been directed to reduce their energy consumption because of an EEPS.

\section{California}

Goals of combined electricity savings of 26,508 GWh by 2013 for PG\&E, SCE and SDG\&E were established in March 2004. This translates into per capita electricity savings of .3\% 
annually.

http://www.cpuc.ca.gov/PUBLISHED/FINAL_DECISION/40212-02.htm\#P123_13438

\section{Hawaii}

Hawaii's renewable portfolio standard requires $10 \%$ of electricity sales to be from renewable sources by $12 / 31 / 10,15 \%$ by $12 / 31 / 15$ and $20 \%$ by $12 / 21 / 20$. Quantifiable and verifiable energy-efficiency measures can be counted towards these goals.

http://www.capitol.hawaii.gov/hrscurrent/Vol05_Ch0261-0319/HRS0269/HRS_0269-0091.htm

\section{Illinois}

Illinois passed a resolution in 2005 creating an energy-efficiency portfolio goal (EEPG), which sets targets of load growth reduction of $10 \%$ for $2007-8$, 15\% for 2009-2011, 20\% for 2012-2014 and $25 \%$ for $2015-2017$.

http://www.commerce.state.il.us/NR/rdonlyres/26A736D5-6B18-46CC-90DA-FB900EBA3DDF/0/IllinoisSustainableEnergyPlan.pdf

\section{Nevada}

In 2005, Nevada's renewable portfolio standard was amended to allow utilities to receive credit towards their renewable goals from energy-efficiency measures. Energy efficiency may represent no more than one-quarter of the total standard in any given year, however. In 2007 and 2008, $9 \%$ of a utility's portfolio must come from renewable energy or energy efficiency, $12 \%$ in 2009 and 2010, 15\% in 2011 and 2012, 18\% in 2013 and 2014 and 20\% from 2015 and later.

http://www.puc.state.nv.us/Renewable/REPSNevada_files/frame.htm

\section{Pennsylvania}

Pennsylvania enacted an Alternative Energy Portfolio Standard in 2004 that required electric suppliers and distributors to supply $18 \%$ of its electricity using alternative-energy resources by 2020. Demand side management, energy efficiency and load management programs and technologies are among those "alternative energy" resources that can be applied to the standard. Pennsylvania's system is unique, however, in that it breaks up eligible technologies into two categories, each of which must account for a certain percentage of the overall alternative energy portfolio. So, while energy-efficiency measures are not capped, at least $8 \%$ of a utility’s 
portfolio in 2020 must come from renewable energy sources including wind, solar PV and geothermal among several others. At least $10 \%$ must come from energy-efficiency measures, distributed generation systems, large-scale hydro and several others.

http://www.puc.state.pa.us/electric/electric_alt_energy.aspx

\section{Texas}

Starting in 2002, Texas required 38 urban and surrounding counties representing more than $70 \%$ of Texas' population to reduce electricity consumption by 5\% each year through 2007 . The bill was enacted to help Texas comply with the Clean Air Act.

http://www.texasenergypartnership.org/g/index.asp

\section{Data Center-Related Tax Incentives}

\section{Maryland}

A green buildings tax incentive program was implemented in 2001, which closely mirrors the 2000 New York green building tax incentive program. Buildings that are 35\% more efficient than current standards and meet state specified criteria are eligible for an income tax credit of up to $\$ 120$ per square foot.

http://mlis.state.md.us/2001rs/billfile/hb0008.htm

\section{Nevada}

In 2005, Nevada passed a law offering a partial exemption from the property tax of up to 50\% for up to 10 years for buildings achieving LEED-silver ratings. Furthermore, products or materials used to construct a LEED-silver building are exempt from the Nevada sales tax.

http://www.leg.state.nv.us/22ndSpecial/Reports/history.cfm?ID=2546

\section{New York}

An income tax credit has been available since 2000 for owners and tenants of buildings and tenant spaces which meet "green" energy efficiency standards. The credit applies to newly constructed buildings and renovations made to existing buildings. \$25 million was initially allocated for these credits, which are allowed for the taxable years 2001-2009. The credit 
certificates stopped being issued in 2004, but an additional \$25 million was authorized in 2005 for credits to be issued through 2009.

http://www.dec.state.ny.us/website/ppu/grnbldg/index.html

\section{Oregon}

Oregon offers business energy tax credits (BETCs) for energy-efficient investments, including energy-efficient equipment and sustainable buildings. The BETCs are for $35 \%$ of the incremental cost of the energy-efficient investment. Public entities that partner with an Oregon business are also eligible for the credit.

http://egov.oregon.gov/ENERGY/CONS/BUS/BETC.shtml

\section{Public Benefits Funds}

Much like Portfolio Standards, Public Benefits Funds do not necessarily relate directly to data center energy conservation. However, depending on what programs the funds raised through system benefits charges support, some of the money could go to financing data center efficiency improvements.

\section{California}

In September 1996, California created a four-year system benefits charge funded through a nonbypassable wires charge. The fund supports low-income, renewable, energy efficiency, and research and development programs. In August 2000, the system benefits fund received a tenyear extension, until 2010, with adjustment for inflation, and the energy-efficiency portion of its funding was expanded to \$228 million per year.

http://www.cpuc.ca.gov/static/energy/electric/energy+efficiency/ee_funding.htm

\section{Connecticut}

In April 1998, Connecticut passed legislation that established the Connecticut Clean Energy Fund to ensure the advancement of energy-efficient technologies and the development of Connecticut's sustainable energy future. In 2003, the fund had a \$109 million annual budget. Due to budget shortfalls, the fund currently operates on a $\$ 70$ million annual budget. Furthermore, from July 2003 to July 2005, and again from August 2006 through July 2007, \$1 million per month was/will be diverted from the fund to the state's general coffer. Funds are collected through a non-bypassable wires charge. 
http://www.ctsavesenergy.org/

\section{Delaware}

In 1999, Delaware passed the Electric Utility Restructuring Act. Revised in 2000, and again in 2003, the Act includes a Public Benefits Fund formerly called the "Environmental Incentive Fund” and re-titled "The Green Energy Fund.” A systems benefit charge gives the fund roughly \$1.5 million in annual revenue for energy efficiency and renewable energy programs. However, so far the fund, administered by the State Energy Office, has only been used for renewable energy programs. Delaware also has a separate systems benefit charge providing about $\$ 0.8$ million a year for low-income programs.

http://www.legis.state.de.us/LIS/LIS142.NSF/vwLegislation/SB\%2093?Opendocument

\section{District of Columbia}

In March of 2005, the District of Columbia appropriated \$20 million for two-year energyefficiency, renewable and low-income energy programs. The programs are funded by the Reliable Energy Trust Fund, a public benefits fund that is funded by a surcharge on residential PEPCO bills. The minimum surcharge is equal to $\$ .0001$ per $\mathrm{kWh}$, while the maximum surcharge equals \$.002 per kWh. Among other things, the fund has been used to support energy efficiency in small businesses, institutions and non profits, and net metering.

http://www.dceo.dc.gov/dceo/cwp/view,a,3,q,603158,dceoNav,|32974|.asp

\section{Louisiana}

A June 2005 bill requests that the Louisiana Public Service Commission continue to work with the Louisiana Association of Community Action Partnerships to develop and implement an Energy Efficiency Fund.

http://www.legis.state.la.us/billdata/streamdocument.asp?did=312860\%20

\section{Maine}

In 2002, the Maine Public Utilities Commission obtained the authority to develop and implement energy-efficiency programs from the public benefits fund, which is financed through a system benefits charge. At least $20 \%$ of the money raised must go towards efficiency programs for small businesses.

http://www.efficiencymaine.com/ 


\section{Massachusetts}

Massachusetts’ 1997 Electric Utility Industry Restructuring Act requires customers of the electric distribution companies to pay a charge to support energy-efficiency programs for residential, commercial and industrial customers. Each distribution company collects \$0.00025 per kWh from all customers except low-income consumers. The statewide expenditures are near \$125M annually with equitable portions of residential and commercial collections subsidizing the low-income sector. The public-benefit fund currently extends through 2012.

http://www.mass.gov/doer/

\section{Michigan}

Michigan's Low Income and Energy Efficiency Fund was created in 2000, and was originally financed through savings from utility securitization (bonds repaid through charges on utility customer bills), but now financed through a utility surcharge. $25 \%$ of the money is dedicated for grants for energy-efficiency projects regardless of customer class or income level.

http://www.michigan.gov/mpsc/0,1607,7-159-16370_27289---,00.html

\section{Minnesota}

Energy utilities are required to devote a percentage of their operating revenues to energy efficiency projects through a Conservation Improvement Program (CIP). State statute mandates that gas utilities invest 0.5 percent, electric utilities invest 1.5 percent, and electric companies that operate nuclear plants invest 2 percent of their gross operating revenues into energy conservation improvements. The utilities collect these funds by adding a surcharge to their rates. This money is used for efficiency R\&D, rebates, home energy audits, and consumer education. In 2003, investor-owned utilities spent about $\$ 65$ million on these programs, while municipal and cooperative utilities spent a combined \$26 million or so.

http://www.auditor.leg.state.mn.us/Ped/pedrep/0504all.pdf

\section{Montana}

In 1997 Montana created an electric universal systems benefits charge that was extended through 2009 in 2005. Utilities are required to use 2.4 percent of their retail sales revenues in 1995 and put it towards energy-efficiency and renewable energy projects and low-income energy assistance. Utilities can direct those funds to internal EERE projects if they wish to, and largescale customers (with a load of at least 1 megawatt) can also use the funds internally rather than paying the charge. There is also a parallel systems benefits charge for the gas industry. 
http://www.deq.state.mt.us/energy/renewable/taxincentrenew.asp\#69-8-402

\section{New Hampshire}

As part of New Hampshire's electric restructuring law, the Legislature created a system benefits charge (SBC) to fund energy efficiency programs and low-income rate assistance. Commercial projects for new construction and major renovations which can be funded by the SBC include lighting upgrades, occupancy sensors, controls, air conditioning improvements, efficient motors, variable-frequency drives, energy-management systems, and individually customized projects.

http://www.nh.gov/oep/programs/energy/resources.htm

\section{New Jersey}

In 2001, an SBC was added to electric utility bills in New Jersey to fund the Clean Energy Program. The Clean Energy Program provides technical assistance, financial assistance, information and education for all utility customers. For commercial customers, the New Jersey SmartStart Buildings program offers free energy-efficiency support on new construction and additions, renovations, remodeling, and equipment replacement. Approximately 75 percent of the revenue goes to energy efficiency programs, while at least 25 percent must go to renewable energy programs. Annual funding was $\$ 124$ million in 2004, and a projected $\$ 745$ million will be collected from 2005-2008.

http://www.njsmartstartbuildings.com/

\section{New Mexico}

In April of 2005, New Mexico passed the Efficient Use of Energy Act. The bill requires public electric and natural gas utilities to implement cost-effective energy-reduction programs. The programs will be funded through a tariff rider for energy-efficiency and load management programs. The charges on the consumer cannot exceed 1.5 percent of the energy bill or $\$ 75,000$ per year. The act will take effect in 2006.

http://legis.state.nm.us/Sessions/05\%20Regular/bills/house/HB0619.pdf

\section{New York}

In July 1998, New York State implemented a system benefits charge (SBC). The money raised goes to energy efficiency, research and development, and low-income programs. The energy efficiency aspect focuses on market transformation, energy services industry programs, and technical assistance and outreach programs. The SBC was extended through June 30, 2011 in December 2005, and its annual funding was increased from \$150 million to \$175 million, of which \$427 million is projected to go to peak load reduction, energy efficiency, and outreach and education programs. . In addition to the SBC, NYSERDA also administers the New York State 
Renewable Portfolio Standard where a subset of these funds are earmarked for fuel cell research, development, and deployment.

http://www.getenergysmart.org/

\section{Ohio}

Created in 1999, Ohio’s Energy Loan Fund provides incentives for energy efficiency, distributed energy and renewable energy projects. The fund offers grants periodically, and also provides low-interest loans for energy-efficiency improvements for government and commercial customers, among others. The Fund is funded by a system benefits charge to customers of Ohio’s investor-owned utilities, equal to about \$100 million over ten years.

http://www.odod.state.oh.us/cdd/oee/energy_loan_fund.htm

\section{Oregon}

Under Oregon's 1999 electricity restructuring law, a three percent public purpose charge is assessed on retail electric customers. These funds are used to support energy efficiency, renewable energy, and low-income weatherization programs. Two-thirds of the funds collected are devoted to energy-efficiency measures. The efficiency programs supported include incentives for energy-efficiency improvements to new commercial buildings and retrofits to existing commercial buildings, and for energy-efficient products, among others.

http://www.energytrust.org/business/index.html

\section{Rhode Island}

In 1996, Rhode Island created the first public benefits fund in the nation. The fund was created to support demand-side management and renewable energy programs. Initially, one system benefits charge funded both the energy-efficiency and renewable programs, but in 2002 separate surcharges were established, to last through 2012, by which time the program should be selfsustaining. The demand-side management programs, funded through a charge of 0.2 cents (\$0.002) per kilowatt-hour, include residential, commercial and industrial programs, and are run by the electric utilities, subject to approval by the Public Utilities Commission.

http://www.dsireusa.org/library/includes/incentive2.cfm?Incentive_Code=RI04R\&state=RI\&CurrentPageID=1\&RE=0\&EE=1

\section{Vermont}

Efficiency Vermont, an “energy efficiency utility,” was created by the Vermont Public Service Board. It is funded by an energy-efficiency charge on consumer electric bills, similar to a system benefits charge. Efficiency Vermont offers energy- and money-saving programs to consumers that incentivize and assist energy-efficient construction, building design, renovation, appliances, 
lighting and equipment. Its annual budget was $\$ 19.5$ million in 2006 and is expected to rise to \$30.75 million by 2008 .

http://www.efficiencyvermont.org/pages/Business/

\section{Wisconsin}

In 1999, Wisconsin created Wisconsin Focus on Energy, a public-private partnership with the goal of encouraging energy efficiency and renewable energy. $1.2 \%$ of every utility's gross operating revenue is required to be spent on energy-efficiency and renewable energy programs. Large customers can fund their own internal projects with money they would otherwise have to pay towards the fund. Commercial programs include the development of energy action plans, energy-training programs, and incentives to help mitigate the incremental cost of implementation of energy-efficient products.

Currently funded through a system benefits charge, starting July 1, 2007, the public benefits fund programs will be replaced with utility programs, created and funded through contracts with private program administrators. This will prevent the fund from being raided for general government needs; from 2002 to 2006 more than \$108 million was diverted from the PBF to the general coffer.

http://www.focusonenergy.com/ 